\title{
Can a stochastic cusp catastrophe model explain housing market crashes?
}

\author{
Cees Diks ${ }^{1}$ and Juanxi Wang*1,2 \\ ${ }^{1}$ CeNDEF, Amsterdam School of Economics, University of Amsterdam, Roetersstraat 11, 1018 \\ WB Amsterdam, The Netherlands \\ ${ }^{2}$ Department of Accounting and Finance, University of Strathclyde, Scotland G4 0QU, United \\ Kingdom
}

July 4, 2016

\begin{abstract}
Like stock market prices, housing prices often exhibit temporary booms and busts. A possible explanation for the observed abrupt changes is offered by the stochastic catastrophe model. This paper addresses the question whether the catastrophe model can describe and predict the dynamics of housing markets. We fit a stochastic cusp catastrophe model to empirical housing market data for six OECD countries, US, JP, UK, NL, SE and BE. Two different estimation approaches are considered - Cobb's method and Euler discretization. The analysis shows that while Cobb's approach describes the long-run stationary density better, Euler discretization is more tailored for time series, as it provides better one-step-ahead predictions. Proceeding using the Euler discretization method we discuss the dynamics of housing markets in terms of the multiple equilibria cusp catastrophe model. By considering the long-term interest rate as an exogenous variable we obtain new insights into the policy implications of interest rate levels, in particular concerning the stability of housing markets.
\end{abstract}

Keywords: stochastic catastrophe, housing market, instability, multiple equilibria JEL Classification: C13, C53

${ }^{*}$ Corresponding author. E-mail address J.Wang@uva.nl 


\section{Introduction}

The collapse of the U.S. housing bubble in 2007 was followed by a worldwide financial crisis. This tragedy has raised great concerns of housing bubbles among financial regulators and researchers. Like stock market bubbles, housing bubbles in hindsight often can be identified with unjustified increases in housing prices before they crash. Fig. 1 illustrates the bust phase of housing price cycles surrounding banking crises from 1899 to 2008 using real housing prices (Reinhart and Rogoff, 2009). The historical average of the declines from peak to trough is 35.5 percent. A number of countries with major housing crashes are included. For instance, Finland, Colombia, the Philippines and Hong Kong have experienced the most severe real housing prices crashes in the past 25 years. The severity of these crashes amounted to 50 to 60 percent from peak to trough. Notably, these housing price declines have been quite long lived, averaging roughly 6 years. After the housing market crash of Japan in 1992, real housing prices declined for a consecutive 17 year period. In particular, housing price declines are even longer lived than equity price declines. The average historical downturn phase in equity prices lasts 3.4 years, about half of the downturn phase in housing prices (Reinhart and Rogoff, 2009). The International Monetary Fund (IMF) recorded that housing price busts lasted nearly twice as long and led to output losses that are twice as large as for asset price busts (IMF World Economic Outlook, 2003). Moreover, financial crises and recessions are often preceded by housing market crashes (Reinhart and Rogoff, 2009). The credit crisis and the global financial crisis in 2008 are convincing examples. After housing prices declined in the latter half of 2007, the secondary mortgage market collapsed. A complex chain reaction almost brought down the worldwide financial system. Furthermore, housing market bubbles are considered as

leading indicators of financial instability and crises (Davis and Heathcote, 2005). For the above reasons, a good understanding of the instability in housing markets is crucial.

Housing market models have been studied extensively in the literature. Unfortunately, most of the available research in macroeconomics is mainly based on state-of-the art dynamic stochastic general equilibrium (DSGE) models which are based on fundamentals. However, these traditional models have difficulty explaining the observed booms and busts in housing prices. A series of papers by Shiller have argued that the changes in economic fundamentals such as population growth, construction costs, interest rates and real rents did not match up with the observed house price fluctuations (Case and Shiller, 2003; Shiller, 


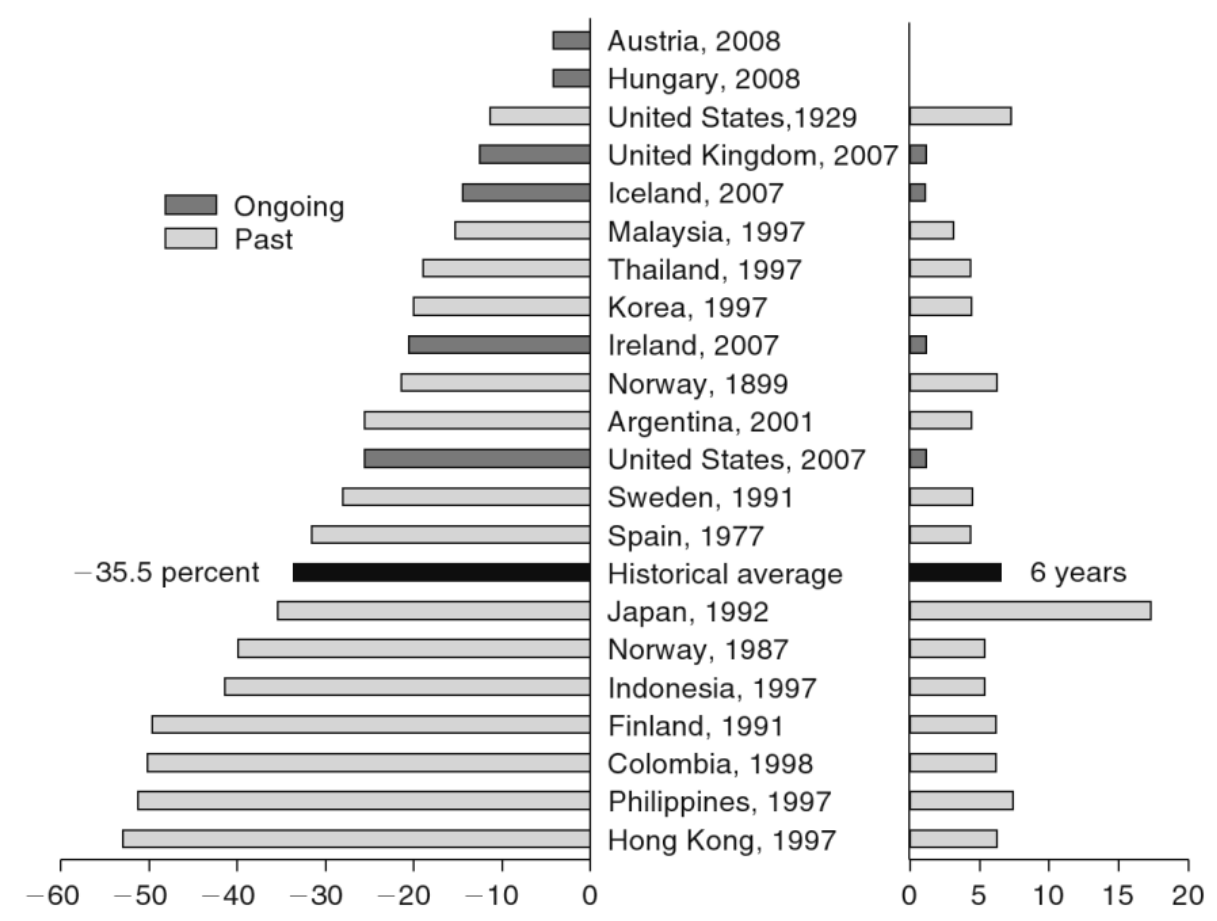

Figure 1: The bust phase of housing price cycles surrounding banking crises. Left panel: peak-to-trough price declines; Right panel: years duration of downturn. [Reproduced with publisher's permission from Reinhart and Rogoff (2009)].

2007, 2008, 2012, 2015). Davis and Heathcote (2005) also suggested that DSGE models with housing consumption and production were unable to capture the instability of house prices.

During the last decades an increasing number of researchers have recognized economic systems as complex systems with multiple equilibria. Recently, a theoretical approach using heterogeneous agent models (HAMs) has been introduced to housing markets, inspired by the work on heterogeneous agent based financial market models; see, for instance, Brock and Hommes $(1997 ; 1998)$ and the comprehensive survey in Hommes (2013)). Kouwenberg and Zwinkels (2015) developed and estimated a HAM model for the U.S. housing market, and showed that the estimated model produces boom and bust price cycles endogenously. Dieci and Westerhoff $(2012,2013,2015)$ also investigated the speculative behavior in housing markets using a HAM approach, identifying a variety of situations leading to irregular endogenous dynamics with long lasting, significant price swings around the fundamental price, like those observed in actual markets. Bolt et al. (2014) established and estimated a 
HAM model for eight different countries, and found evidence of heterogeneous expectations from empirical data and identified temporary house price bubbles for various countries.

Although HAMs turned out to be successful theoretical tools to capture temporary deviations from market equilibrium, a related statistical time series analysis method is still lacking. In this paper, we therefore consider and compare various statistical methods to describe the price dynamics governing real housing price time series. Catastrophe theory has been suggested to be a good candidate approach for describing (parts of) the economy (Zeeman, 1974); it can capture the inherent instability in many nonlinear dynamical systems and has proven to be an extremely successful tool to investigate the qualitative properties in a wide range of different complex systems, ranging from physics and engineering to biology, psychology and sociology. Its applications involve urban and regional systems (Wilson, 1981), quantum morphogenesis (Aerts et al., 2003), the stability of black holes (Tamaki et al., 2003), the size of bee societies (Poston and Stewart, 2012), the cognitive development of children (Van der Maas and Molenaar, 1992), sudden transitions in attitudes (Van der Maas et al., 2003) and so on. In all these applications, the behavior of the observed system shows sudden and discontinuous changes or critical transitions as a result of a small change in one or more control variables. Catastrophe theory offers a mathematical basis for the number and the type of critical points for the classification of nonlinear dynamical systems. Since the economic system has become widely recognized as a complex system (see e.g. the special issue of JEDC on Complexity in Economics and Finance, edited by Anufriev and Branch (2009)) displaying quick transitions such as market crashes, catastrophe theory might be a good candidate to explain its extreme fluctuations. Zeeman (1974) already proposed that some of the unstable behavior of stock exchanges could be explained by a model based on catastrophe theory. A similar model can also be applied to currencies, property markets, or any market that admits speculators. Barunik and Vosvrda (2009) and Barunik and Kukacka (2015) followed Zeeman's suggestion and fitted a stochastic cusp catastrophe model to stock market data. They provided an important shift towards the application of catastrophe theory to stock markets. In particular their examples showed that stock market crashes were better explained by cusp catastrophe theory than other models.

In this paper, following up on the idea of Zeeman (1974), we fit a stochastic cusp catastrophe model to housing market data. To the best of our knowledge this has not been done before. The aim of this paper is twofold. Firstly, we compare two estimation 
methods: Cobb's method and Euler discretization. We show that Euler discretization gives better forecasting ability than Cobb's method in a time series context. Secondly, we obtain insights into the underlying mechanisms of the instability of housing markets by interpreting the estimated cusp catastrophe models. We also investigate the link between interest rates and housing price dynamics.

Our analysis sheds some light on the application of catastrophe theory to time series data in social science. We fit a stochastic cusp catastrophe model to housing markets in six different countries: The United States (US), Japan (JP), The United Kingdom (UK), The Netherlands (NL), Sweden (SE) and Belgium (BE). Our results show how the number and the stability of the equilibria of the system can change depending on the interest rate for each country. This scenario can be used to explain several housing price bubbles and crashes in empirical data, such as UK 1978, 1980, 1990, NL 1978, 1990, and the depression of SE after 1990. The main policy implication of our study is that policy makers should adopt an interest rate policy that prevents the system from getting too close to the cusp curve that may induce a systemic market crash. To achieve this, the cusp catastrophe fit could provide a reasonable guidebook.

This paper is organized as follows. We first introduce, in Section 2, the cusp catastrophe theory and its application to housing markets. Subsequently, we discuss the empirical methods of Cobb and Euler discretization, estimation variables and empirical data in Sections 3 and 4 . The results of the Euler discretization approach are presented and discussed in Section 5. We provide a number of concluding remarks in Section 6.

\section{Catastrophe Theory}

Catastrophe theory has been first proposed by the French mathematician René Thom (1972). Before his work, most models only described phenomena with smooth and continuous changes. However, the world is full of sudden irregularities and unpredictable transitions. The proposed catastrophe theory has shed some light on the underlying "laws of nature". Zeeman cooperated with Thom and proposed catastrophe theory applications in the fields of economics, psychology, sociology, political studies, and others (Zeeman, 1974, 1977). In particular, he proposed the application of the cusp catastrophe model to stock markets and qualitatively described the bull and bear markets as a result of interaction between two main types of investors: fundamentalists and chartists (Zeeman, 1974). 
This work contains a number of important behavioral finance elements, which later led to research on HAM models. However, the biggest difficulty in the application of catastrophe theory arises from the fact that it was developed for deterministic systems, while most scientific contexts require one to allow for at least some random noise. In order to apply it directly to behavioral science, where random influences are common, a bridge between deterministic and stochastic catastrophe theory is needed. Loren Cobb (1981a) addressed this challenge by developing a stochastic version of catastrophe theory based on stochastic differential equations (SDEs).

The stochastic cusp catastrophe model corresponds to a specific parametric SDE, that allows for a cusp bifurcation with a multiple equilibria parameter region and corresponding critical transitions. Barunik and Vosvrda (2009) and Barunik and Kukacka (2015) fitted the stochastic cusp catastrophe model to stock market data and showed that it could explain stock market crashes better than linear models. As noted in the Introduction, housing market crashes often take place prior to financial crises and recessions, which is why housing bubbles are considered as leading indicators of financial instability and crises. To the best of our knowledge there are no existing cases of housing market analyses based on catastrophe theory. Therefore, our main goal is to use catastrophe theory to model the dynamical behavior in housing markets.

Catastrophe theory provides a mathematical basis for describing systems involving discontinuous and divergent phenomena. In particular, it is effective for describing systems where gradually changing forces can lead to abrupt changes in behavior. It is based on a single variable nonlinear dynamical system, which follows, in the noise-less case, the differential equation

$$
\mathrm{d} y_{t}=-\frac{\partial V\left(y_{t} ; \theta\right)}{\partial y_{t}} \mathrm{~d} t
$$

where $y_{t}$ represents the state of the system. This implies that the system changes in response to a change in $V\left(y_{t} ; \theta\right)$, where $V\left(y_{t} ; \theta\right)$ is a potential function which is determined by a control parameter $\theta$ determining the specific structure of the system. The system is in equilibrium when the spatial derivative of the potential function equals 0 , i.e. $\mathrm{d} V\left(y_{t} ; \theta\right) / \mathrm{d} y_{t}=0$. The equilibrium corresponds either to a maximum or a minimum of the potential function $V(y ; c)$ with respect to $y$. When $V\left(y^{*} ; \theta\right)$ is a (local) minimum, the equilibrium point $y^{*}$ is stable; the system will return to it after a small perturbation with respect to system's state. Likewise, an equilibrium point $y^{*}$ is unstable if the potential 
function $V\left(y^{*} ; \theta\right)$ corresponds to a (local) maximum. In that case even a small perturbation will drive the system away from the unstable equilibrium state. The local stability properties of these equilibria can change when control variables affecting $\theta$ change, which is why these systems can give rise to unexpected bifurcations when the control variables change. Therefore, catastrophe theory can be employed in systems in which equilibrium states can be driven towards instability, such as gradient dynamical systems with critical points.

\subsection{Cusp Catastrophe}

One of the findings of catastrophe theory is that the behavior of deterministic dynamical systems around the critical points of potential function $V\left(y_{t} ; \theta\right)$ can be characterized by a set of seven canonical forms, with no more than four control variables and one or two canonical state variables (Thom,1972; Zeeman, 1976; Gilmore,1993). In behavioral sciences, the most commonly used canonical form is the so-called cusp catastrophe. In terms of a normalized variable $z_{t}$, it describes sudden, discontinuous transitions in the equilibrium states depending on two control parameters $\alpha$ and $\beta$, within the normal form of the system given by

$$
V\left(z_{t} ; \alpha, \beta\right)=\frac{1}{4} z_{t}^{4}-\frac{1}{2} \beta z_{t}^{2}-\alpha z_{t} .
$$

The equilibria correspond to the solutions to the cubic equation

$$
-\frac{\partial V(z ; \alpha, \beta)}{\partial z}=-z^{3}+\beta z+\alpha=0 .
$$

For descriptive purposes, Cobb (1981b) proposed to use Cardan's discriminant, defined as

$$
\delta=27 \alpha^{2}-4 \beta^{3},
$$

to distinguish the case of three solutions from the case of one solution; for $\delta<0$ there are three equilibria, while there is only one for $\delta>0$. Fig. 2 provides a visualization of the equilibria within the cusp catastrophe model. It shows the cusp equilibrium surface in a three dimensional space. The folded surface with a cusp fold represents the equilibrium surface of the system. The floor is a two dimensional control plane which is determined by a set of control parameters, $\alpha$ and $\beta$. The height "predicts" the value of the system's equilibrium state given the values of the control parameters. 


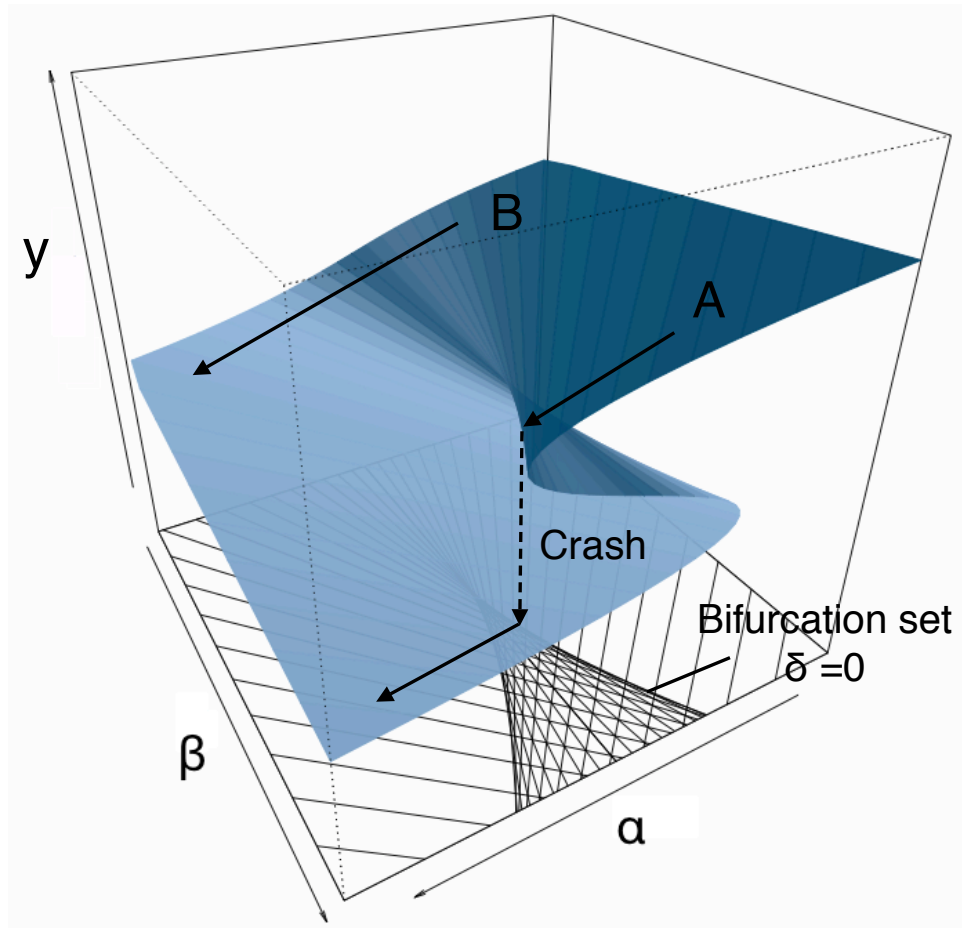

Figure 2: Equilibria in the cusp catastrophe model. [Figure produced using the cusp R package].

The center of the graph shows two sheets representing the behavior of the system, connected by a sheet in the middle that makes it into a continuous, folded, surface. The difference between the middle sheet and the other two sheets is that the middle sheet represents the unstable equilibrium of the system, and the other two stable equilibria. The curve defining the edges of the fold cusp projected onto the control plane give a cusp shaped region. The cusp which marks its boundary is called the bifurcation set (Zeeman, 1974, 1976), for which Cardan's discriminant $\delta$ is zero. For $\delta>0$, the system has only one stable equilibrium state. There is only one predicted state value. However, within the cusp fold area, $\delta<0$, and the surface predicts two possible stable state values instead of one. Moreover, as the system parameters vary, the system might show hysteresis. The state can jump between the two possible state values, but the jump from the top sheet to the bottom sheet requires a different value of the control parameter than the jump from the bottom to the top sheet. 


\subsection{Stochastic Cusp Catastrophe}

Although Zeeman 1977 suggested that catastrophe theory could be applied to multiple disciplines, a practical empirical investigation requires a model that allows for stochastic shocks. In order to address this, and to build a bridge between catastrophe theory and real scientific data, several stochastic formulations of catastrophe theory that allow empirical investigations have been proposed (Oliva et al., 1987; Guastello, 1988; Alexander et al., 1992; Lange et al., 2000). Among the various methods, that of Cobb and Watson (1980) is usually considered the most appealing. They proposed to combine deterministic catastrophe theory with stochastic systems theory by using stochastic differential equations (SDEs). It leads to the definitions of stochastic equilibrium state and stochastic bifurcation that are compatible with their deterministic counterparts, so that a link between the potential functions of deterministic catastrophe systems and the stationary probability density functions (PDFs) of stochastic processes is established.

Assuming that the (canonical) variable $z_{t}$ is governed by the potential function of Eq. (2), and that there is a driving noise term with variance $\sigma_{z}^{2}$ per time unit, the dynamics can be written in terms of the stochastic differential equation (SDE)

$$
\begin{aligned}
\mathrm{d} z_{t} & =-\frac{\partial V\left(z_{t} ; \alpha, \beta\right)}{\partial z_{t}} \mathrm{~d} t+\sigma_{z} \mathrm{~d} W_{t} \\
& =\left(-z_{t}^{3}+\beta z_{t}+\alpha\right) \mathrm{d} t+\sigma_{z} \mathrm{~d} W_{t}
\end{aligned}
$$

where $-\partial V\left(z_{t} ; \alpha, \beta\right) / \partial z_{t} \mathrm{~d} t$ is the drift term, $\sigma_{z}$ is the diffusion parameter, $W_{t}$ represents a Wiener process, $z_{t}$ is the dependent variable, and $\alpha$ and $\beta$ are the "canonical variates" which are assumed to be smooth functions of $v$ observable exogenous control variables $x_{1}, \ldots, x_{v}, v=0,1, \ldots$

Since for $\alpha=0$ the stationary PDF of $z_{t}$ is symmetric and the sign of $\alpha$ determines whether it is left or right skewed, $\alpha$ is called the "asymmetry factor". If $\beta$ is increased sufficiently, the stationary density changes from unimodal to bimodal. Therefore $\beta$ is called the "bifurcation factor" determining the number of modes of the density.

\subsection{Cusp Catastrophe Behavior of Housing Markets}

The best way to understand the nature of the cusp catastrophe model is to illustrate it by examples. Zeeman has considered some popular applications of the cusp catastrophe model in a number of different disciplines, including ecology, physics and psychology (Zeeman, 
1974). His work was the first attempt to explain the unstable behavior of a stock market using the catastrophe model. The housing market has several common characteristics with the stock market. Their dynamical behaviors are connected in some ways. The boom and bust cycle of housing prices has also been found to be at least partly driven by heterogeneous expectations of agents (Bolt et al., 2014). Several theoretical models have been shown to perform well in the analysis of housing markets and stock markets. For instance, HAMs are successful in capturing the instability in both the stock market and the housing market (Brock and Hommes, 1997, 1998; Hommes, 2013; Kouwenberg and Zwinkels, 2015; Dieci and Westerhoff, 2012, 2013, 2015; Bolt et al., 2014). Finally, since Zeeman has suggested that the catastrophe model could be used to model financial markets, it might also be useful in the housing market.

For the housing market, the cusp catastrophe model represented visually in Fig. 2 can be interpreted as follows. The $z$-axis of the 3 -dimensional space, $y$, represents the state variable. In our analysis this will be the housing price level in terms of its relative deviation from the fundamental price. The folded surface with three levels of sheets represents the three equilibrium prices of the system. The set of control parameters $\alpha$ and $\beta$ forms a two-dimensional control plane.

Critical transitions can now occur when the control parameters make the state move across the multiple equilibrium area of the cusp equilibrium surface. Each point on the top and bottom sheets of this surface gives an equilibrium of the system. If the state is on the top sheet and follows path $A$ on the control surface, the corresponding path moves to left on the top sheet until it reaches the fold curve; the top sheet then vanishes, and the path will suddenly jump to the bottom sheet. In this way, a small change in control parameters can produce a sudden large change in the state of the system. Alternatively, path $B$ on the control surface outside of the cusp bifurcation exhibits the behavior of a more tranquil market. Its corresponding path moves to the bottom sheet slowly and smoothly, without critical transitions. A similar mechanism of boom-bust cycles has recently been described by Dieci and Westerhoff (2015) in an expectations-driven dynamical context.

The mechanism of housing market crashes can be understood in terms of the cusp catastrophe model as follows. Suppose that the control parameters are such that the housing market has multiple equilibria, and the market is on the top sheet of the equilibria surface. That is, the market state is in the high price equilibrium, while there is another, low price, stable equilibrium on the bottom sheet of the equilibrium surface. A crash 
can then be induced by any event that changes the control parameters just enough to push the state variable over the fold curve, so that it falls off the "cliff" and jumps to the bottom sheet. In particular, if the state is in the upper stable equilibrium within the multiple equilibria region and very close to the cusp curve, even a small perturbation can induce large market collapse. Similarly, "negative" crashes (booms) could be induced by an upward jump from the bottom sheet to the top sheet. Additionally, due to exogenous disturbances (noise) the system could also transition from one stable equilibrium to another without passing through the cusp curve. The cusp catastrophe model can also explain the slow recovery from a crash in housing markets. The recovery is affected by hysteresis; simply reversing the control parameters back does not have major effects until the control parameters exit the multiple equilibria region again. An alternative route to recovery is obtained by smoothly following the reversal of path $B$.

\section{Estimation Methods}

In the estimation of the cusp catastrophe model, the control parameters $\alpha$ and $\beta$ are usually assumed to be (well aproximated by) linear functions of the exogenous variables $x_{1}, \ldots, x_{v}$, that is,

$$
\begin{aligned}
\alpha & =\alpha_{0}+\alpha_{1} x_{1}+\alpha_{2} x_{2}+\ldots+\alpha_{v} x_{v} \\
\beta & =\beta_{0}+\beta_{1} x_{1}+\beta_{2} x_{2}+\ldots+\beta_{v} x_{v} .
\end{aligned}
$$

We will follow this commonly made assumption. The canonical dependent state variable $z$ is also assumed to be a linear function of or more observable dependent state variables $y_{1}, \ldots, y_{k}$ of the system, that is,

$$
z=w_{0}+w_{1} y_{1}+w_{2} y_{2}+\ldots+w_{k} y_{k}
$$

where $w_{0}, w_{1}, \ldots, w_{k}$ are coefficients.

Fitting the cusp model to empirical data thus involves estimating the coefficients $w_{0}, w_{1}, \ldots, w_{k}, \alpha_{0}, \alpha_{1}, \ldots, \alpha_{v}$ and $\beta_{0}, \beta_{1} \ldots, \beta_{v}$. In our applications we will only consider cases where $v=0$ (constant control parameters) or $v=1$ (both control parameters being linear functions of the interest rate), and $k=1$ (a single dependent variable $y$ ) $z=w_{0}+w_{1} y$, or, reparameterized, $z=(y-\lambda) / \sigma$, where $\lambda$ is a location parameter and $\sigma$ a scale parameter. 


\subsection{Cobb's Method}

The most common estimation method that allows quantitative comparison of catastrophe models with empirical data is that proposed by Cobb (Cobb, 1978, 1981a, 1981b; Cobb and Watson, 1980; Cobb et al., 1983). He established a stochastic cusp catastrophe by simply introducing a stochastic Gaussian white noise, representing the noise term in the SDE given in Eq. (5).

In this approach, maximum likelihood estimation is applied using the cusp PDF. To simplify the estimation procedure, instead of using the conditional PDF $f\left(z_{t} \mid z_{t-1}, z_{t-2}, \ldots ; \alpha, \beta\right)$, Cobb considered the stationary PDF which is given by solving the corresponding FokkerPlanck equation. As time $t$ increases, the conditional PDF $f\left(z_{t} \mid z_{t-1}, z_{t-2}, \ldots ; \alpha, \beta\right)$ converges to a stationary and time invariant form $f\left(z_{t} \mid \alpha, \beta\right)$. Upon using the linear transformation of the dependent variable, $z_{t}=\left(y_{t}-\lambda\right) / \sigma$, the stationary distribution of the system's state variable $y$ can be expressed as

$$
f_{Y}(y)=\psi \exp \left[\tilde{\alpha}\left(\frac{y-\lambda}{c}\right)+\frac{1}{2} \tilde{\beta}\left(\frac{y-\lambda}{c}\right)^{2}-\frac{1}{4}\left(\frac{y-\lambda}{c}\right)^{4}\right],
$$

where $\tilde{\alpha}=\left(\frac{\sigma_{z}^{2}}{2}\right)^{-\frac{3}{4}} \alpha$ and $\tilde{\beta}=\left(\frac{\sigma_{z}^{2}}{2}\right)^{-\frac{1}{2}} \beta, c=\sigma\left(\frac{\sigma_{z}^{2}}{2}\right)^{\frac{1}{4}}$, and $\psi$ is a normalization constant. For a derivation of the this, see Appendix A.1.

Assuming a random sample, the relevant theoretical PDF to be used in the estimation is the stationary PDF. The stable and unstable equilibria associated with the potential function turn out to correspond to the modes and anti-modes of the stationary PDF, respectively. Such a correspondence does not hold in general, but results from the SDE (5) being homogeneous, that is, the diffusion parameter $\sigma_{z}$ does not depend on $z$ (see e.g. Wagenmakers et al., 2005). The unstable middle sheet of the equilibrium surface in the stochastic case corresponds to the low probability mode between the two high probability modes. The stochastic bifurcations correspond to changes in the number and type of the modes of the stationary PDF. As noted above, a qualitative change in the potential function is identical to a qualitative change in the PDF with respect to the change of control parameters. This is why, for instance, the PDF changes from unimodal to bimodal as the bifurcation parameter $\beta$ increases.

Based on Cobb's statistical catastrophe theory Hartelman (1997), Hartelman et al. (1998), and Grasman et al. (2009) implemented and extended Cobb's estimation method. They presented robust and practical software which made it easy to fit cusp catastrophe 
models on empirical data statistically. In these approaches, Cobb's method is combined with the subspace fitting method of Oliva et al. (1987).

Although Cobb's method has proved to be a successful tool for estimating several multiple equilibria systems, the assumption that the observations are obtained as a random sample from the stationary distribution are quite restrictive. For this to be a reasonable assumption in practice requires a system in which the state variable of interest changes much more quickly than the control parameters. This method gives a good fit of the overall stationary density for a cross-sectional data set, or for those systems with quickly changing state variables relative to control parameters. For typical time series data containing serially dependent observations, or for systems in which the control parameters change relatively fast compared to the state of the system, so that the state variable does not have sufficient time to converge to the stationary distribution, the forecasting ability of Cobb's method would be poor.

\subsection{Estimation Using Euler Discretization}

In order to estimate the stochastic differential equation (SDE) of a cusp catastrophe from time series observations, we consider an alternative numerical method - Euler discretization, which seeks to integrate the evolution determined by SDE over finite time intervals, and thus to arrive at a discrete time approximation of the dynamics. The time variable is subdivided into intervals of length $\Delta t$. Then we can approximate the solution at the corresponding discrete times. In terms of the rescaled variable $y_{t}=\lambda+\sigma z_{t}$ the SDE becomes (see Appendix A.1)

$$
\frac{1}{\sigma} \mathrm{d} y_{t}=-\left.\frac{\partial V(z ; \alpha, \beta)}{\partial z}\right|_{z=\frac{y_{t}-\lambda}{\sigma}} \mathrm{d} t+\sigma_{z} \mathrm{~d} W_{t} .
$$

Euler discretization leads to an approximate equation for the state $y_{t+\Delta t}$ in terms of the current state $y_{t}$, given by

$$
\begin{aligned}
y_{t+\Delta t} & =y_{t}-\left.\frac{\partial V\left(z_{t} ; \alpha, \beta\right)}{\partial z_{t}}\right|_{z_{t}=\frac{y_{t}-\lambda}{\sigma}} \sigma \Delta t+\sigma \sigma_{z} \sqrt{\Delta t} \varepsilon_{t+\Delta t}+\text { h.o.t. } \\
& \approx y_{t}+\left(\alpha+\beta\left(\frac{y_{t}-\lambda}{\sigma}\right)-\left(\frac{y_{t}-\lambda}{\sigma}\right)^{3}\right) \sigma \Delta t+\sigma \sigma_{z} \sqrt{\Delta t} \varepsilon_{t+\Delta t}
\end{aligned}
$$

where $\varepsilon_{t+\Delta t} \sim N(0,1)$. The idea is to use this as a regression equation by which the unknown parameters can be estimated (Florens-Zmirou, 1989; Yoshida, 1992). However, 
the model is over-parameterized in the sense that the forecasts it produces are independent of the units in which we measure $\Delta t$. We therefore set $\Delta t=1$ throughout without loss of generality (i.e. we define one time unit to correspond to one quarter, the time interval between consecutive observations in our data set).

Due to the nonlinear dependence of the conditional mean on the parameters, the parameters are estimated using nonlinear least squares (NLS), using the function ' $n l s()$ ' in R. Although we could alternatively estimate the potential function using ordinary least squares (OLS) regression (Brillinger, 2007), the coefficients of the estimated model would then be nonlinearly related to $\alpha, \beta, \lambda$ and $\sigma$. Using NLS directly provides us with estimates

as well as standard errors of these parameters that can be readily interpreted in terms of the cusp mcatastrophe model.

Finally, we note that during estimation the location parameter $\lambda$ is kept fixed at 0 since there is a fundamental equilibrium at 0 (and the mean of the price fluctuations around this is assumed to be zero). We found this to stabilize the estimation procedure without affecting the goodness of fit by much.

\subsection{Cobb's Method v.s. Euler Discretization}

Intuitively, Euler discretization could offer a considerable improvement to Cobb's method regarding the forecasting ability in a time series setting, where the observations are made on time scales on which the system is varying only slowly, as might be the case for quarterly housing price data. To compare the forecasting ability of the two estimation approaches, a simple and straightforward way is to examine their residuals. Although the data description and specification of the variables will be given only in Section 4, we here give a brief impression of the different nature of the residuals for the two methods here.

Fig. 3 shows the plots of residuals against time in the case of the US by using Cobb's Method and Euler discretization respectively. Fig. 3 (a) shows strongly correlated residuals for Cobb's method, with clear patterns and obvious deviations from randomness. Moreover, the values of the residuals are relatively large, and widespread, in cases being larger than 0.1 in absolute value. This is due to the fact that the predicted values in Cobb's method are estimated based on the closest maximum mode of the stationary PDF rather than by past information. As Fig. 3 (b) shows, the residuals for the Euler discretization approach are much more randomly distributed and less widely spread, with values smaller than 0.04 


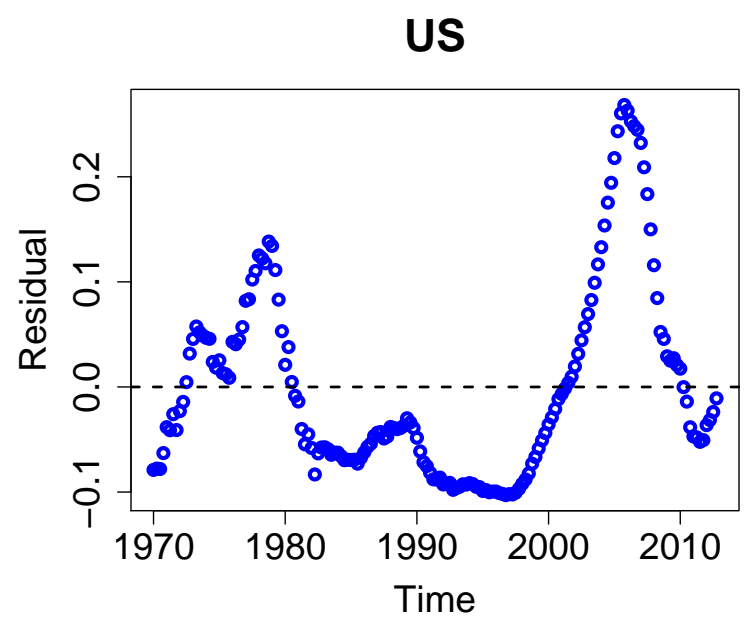

(a) Cobb's Method

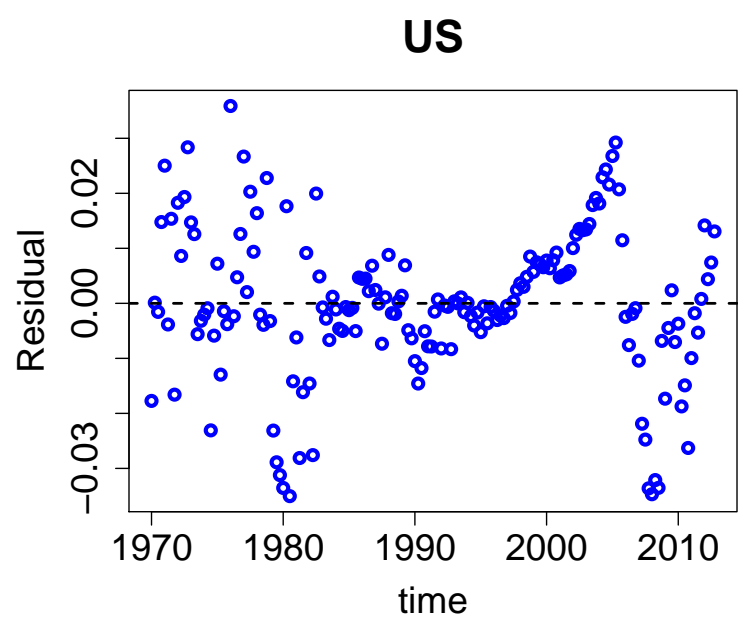

(b) Euler discretization

Figure 3: Time series of residuals obtained using Cobb's Method and Euler discretization in the example of US.

in absolute value. This indicates that indeed Euler discretization gives much better onestep-ahead predictions than Cobb's method. The residuals in the examples of the other countries, shown in Figs 11 and 12 in Appendix A.2, show similar patterns.

Table 1: AIC and BIC of Cobb's method and Euler discretization for different countries.

\begin{tabular}{r|c|cccccc}
\hline & & US & JP & UK & NL & SE & BE \\
\hline \multirow{3}{*}{ Cobb } & AIC & 497.955 & 491.587 & 438.482 & 357.619 & 361.697 & 379.087 \\
& BIC & 510.568 & 504.200 & 451.096 & 370.232 & 373.258 & 391.049 \\
\hline \multirow{2}{*}{ Euler } & AIC & -983.611 & -901.021 & -715.484 & -721.421 & -592.250 & -782.153 \\
& BIC & -971.021 & -888.431 & -702.894 & -708.831 & -580.719 & -770.191 \\
\hline
\end{tabular}

Hartelman (1997) and Grasman et al. (2009) proposed to use Akaike's Information Criterion (AIC) and the Bayesian Information Criterion (BIC) to assess the model fitness. The AIC and BIC in our examples are presented in Table 1. It can be seen that the AIC and BIC by using Euler discretization are much smaller than using Cobb's method, which suggests a better model fit using Euler discretization. It further proves that the one-step- 
ahead forecasting ability of Euler discretization is more promising than Cobb's Method with respect to housing prices.

From these results we conclude that Euler discretization is better able to capture the dynamics than Cobb's method. Therefore, in what follows, we will analyse the cusp catastrophe behavior of housing markets using the Euler discretisation estimation approach.

\section{Estimation Variables}

We consider the housing markets of six different countries: US, UK, NL, JP, SE and BE. The variables involved in the estimation of the cusp catastrophe model consist of state variables and control variables.

\subsection{State Variables}

A state variable is required to be able to describe the unstable behavior of housing markets.

Bolt et al. (2014) estimated a HAM model for housing markets in different countries and observed bifurcations driven by a policy parameter (the interest rate) in the dynamics of the relative deviation of the housing price from the fundamental price. Following these authors, we also use the relative deviation of housing price from the estimated fundamental price as state variable, which is denoted by

$$
y_{t}=\frac{p_{t}}{p_{t}^{*}}-1 \approx \ln p_{t}-\ln p_{t}^{*} .
$$

The fluctuations of the housing price around the fundamental price (based on expected future rental prices) are described by a model in which agents choose between either buying or renting house. In this model, agents make their decisions at time $t$ based on the expected excess return on investing in housing relative to renting during the period between time $t$ and $t+1$. The fundamental price is assumed as the price that would prevail under rational expectations about the conditional mean of the next excess return. In equilibrium, the annual cost of home ownership must equal the housing rent adjusted for risk. The supply of the market is the stock of housing. The demand of agents is determined by maximizing one-period ahead expected excess returns adjusted for risk. By solving the market clearing condition for the price $p_{t}$ (see Bolt et al. (2014) for a detailed calculation), we obtain the 
price equation

$$
p_{t}=\frac{1}{1+r+a} \mathbb{E}_{t}\left[p_{t+1}+\left(1+r^{\mathrm{rf}}\right) Q_{t}\right],
$$

where $Q_{t}$ denotes the price for renting one unit of housing in the period between times $t$ and $t+1, r$ is the sum of the risk free mortgage rate $r^{\mathrm{rf}}$ and the maintenance/tax rate, and $a$ is interpreted as a risk premium of buying a house over renting a house.

The fundamental rental price level $Q_{t}$ underlying the model is assumed to follow a geometric Brownian motion with drift (cf. Boswijk et al. (2007)):

$$
\ln Q_{t+1}=\mu+\ln Q_{t}+v_{t+1}, \quad\left\{v_{t}\right\} \stackrel{i . i . d .}{\sim} N\left(0, \sigma_{v}^{2}\right) .
$$

When $g=e^{\mu+1 / 2 \sigma_{v}^{2}}-1$ and $\eta_{t+1}=e^{v_{t+1}-1 / 2 \sigma_{v}^{2}}$, one obtains

$$
\frac{Q_{t+1}}{Q_{t}}=(1+g) \eta_{t+1},
$$

such that $\mathbb{E}_{t}\left(\eta_{t+1}\right)=1$. By applying the law of iterated expectations and imposing the transversality condition, the fundamental price at time $t$ is found to be

$$
p_{t}^{*}=\mathbb{E}_{t}\left[\sum_{i=0}^{\infty} \frac{\left(1+r^{\mathrm{rf}}\right) Q_{t+i}}{(1+r+a)^{i+t}}\right]=\frac{1+r^{\mathrm{rf}}}{r+a-g} Q_{t}, \quad r+a>g .
$$

This shows that the fundamental price of housing is directly proportional to the actual rent level. For details on identifying the parameters in the proportionality factor, which cannot all be estimated simultaneously, we refer the interested reader to Bolt et al. (2014). Fig. 4 shows an example of house price and fundamental price in the US from 1970 to 2013. Fig. 4(a) presents the housing price index $p_{t}$ with the corresponding estimated fundamental values $p_{t}^{*}$. The relative price deviation $\left(p_{t}-p_{t}^{*}\right) / p_{t}^{*}$, which we will use as the state variable $y$ here, is shown, for the US, in Fig. 4(b). Plots for the other countries are presented in Fig. 13 in Appendix B.

\subsection{Control Variables}

Since we wish to keep the model stylized and simple to interpret we will allow for only one control variable. Taking into account a control variable might help us to understand the role of the control variable in housing market crashes.

One of the parameters which has the greatest influence on the deviations of housing prices from fundamentals is argued to be the long term interest rate. Several authors have 


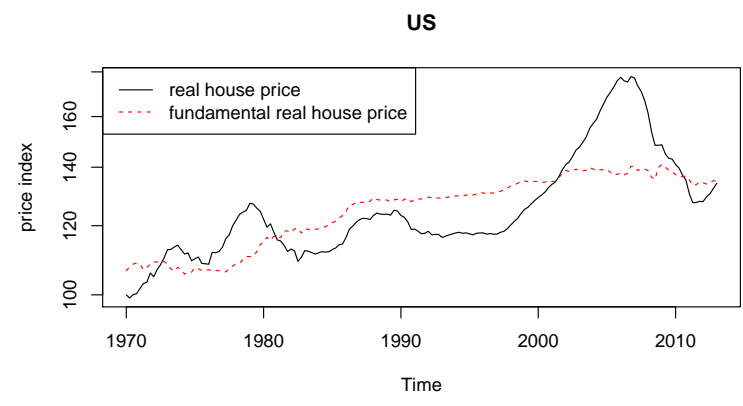

(a) $p_{t}$ and $p_{t}^{*}$

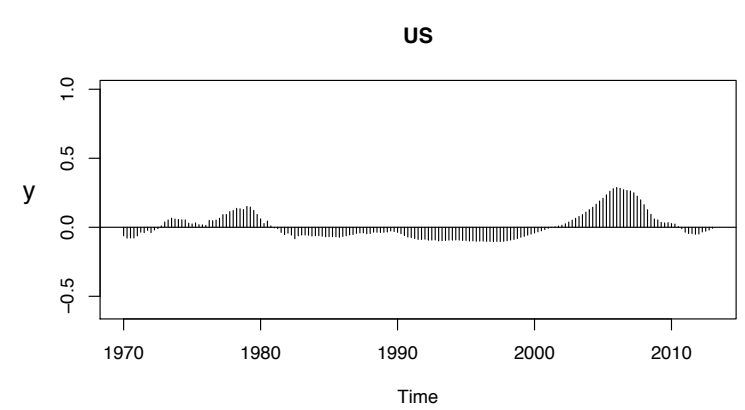

(b) $y_{t}=p_{t}-p_{t}^{*}$

Figure 4: Example of housing price and fundamental price for the US from 1970 to 2013. (Source: Bolt et al., 2014). (a) The housing price index $p_{t}$ with the corresponding estimated fundamental value $p_{t}^{*}$; (b) The relative difference between the two, $\left(p_{t}-p_{t}^{*}\right) / p_{t}^{*}$.

pointed out that monetary policy, especially interest rate has a great impact on housing prices (Bernanke and Gertler, 1995; Shiller, 2006; Taylor, 2007; Muellbauer and Murphy, 2008; Crowe et al., 2013; Shi et al., 2014). Taylor (2009) argued that the interest rate policy was responsible for accelerating the housing boom and thereby ultimately leading to the housing bust. He provided empirical evidence that the unusually low interest rate policy was a factor in the housing boom during 2008 financial crisis. Moreover, empirical evidence suggests that several bifurcations involving price equilibria may occur driven by interest rates (Bolt et al., 2014). Therefore, as an important policy parameter, the long term interest rate is chosen as our control variable.

\subsection{Data Description}

The housing markets considered are those of the US, UK, NL, JP, SE and BE. In order to estimate the model parameters we require time series as long as possible to use as much information as possible. The investigated time window ranges from the earliest available year 1970 to $2013^{1}$. This contains several well-known housing market crashes, such as those in the United States (2007), Japan (1992), Sweden (1991), the United Kingdom (2007) and on-going bubbles in many countries.

Quarterly nominal and real house prices for each country are obtained from the Organ-

\footnotetext{
${ }^{1}$ The time window for Sweden (SE) is from 1980Q1 to 2013Q1, and for Belgium is from 1976Q2 to 2013Q1, based on the availability in the datasets.
} 
isation for Economic Co-operation and Development (OECD). The nominal house price is indexed using 2005 as base year. The real house price index is derived by deflating with the private final consumption expenditure deflator, which is available from the OECD Economic Outlook 89 database. The price-to-rent ratio is defined as the nominal house price index divided by the rent component of the consumer price index, made available by the OECD. Interest rates are long-term interest rates that are implied by the prices at which government bonds are traded on financial markets, not the interest rates at which the loans were issued. They refer to bonds whose capital repayment is guaranteed by governments (from the OECD iLibrary database). Therefore, the 10 year government bond yield is considered a standard indicator of long-term interest rates. They are obtained from the OECD iLibrary for US, UK, NL, BE. Because the OECD iLibrary did not have the interest rate dating back to 1970 for JP and SE, those were obtained separately from Datastream.

\section{Empirical Results and Discussion}

The parameters of the differential equation of the cusp catastrophe model, Eq. (5), are estimated using the Euler discretization approach. As described in Section 4, the state variable is the relative deviation of the housing price from the fundamental price. For the control parameters, we consider two variants: estimation with constant control parameters $\alpha$ and $\beta$, and estimation with the control variable interest rate governing the control parameters $\alpha$ and $\beta$.

\subsection{Constant Control Parameters}

As a benchmark, we fit the cusp catastrophe model to the housing market data with a constant control variable. Thus the control parameters $\alpha$ and $\beta$ are constant and according to Eq. (6) are given by $\alpha=\alpha_{0}$ and $\beta=\beta_{0}$. To estimate the stability of the system statistically, we also calculate the implied values of Cardan's discriminant $\delta=27 \alpha^{2}-4 \beta^{3}$. Recall that $\delta=0$ indicates the boundary of the multiple equilibria region, and that when $\delta<0$, the state of the system is in the multiple equilibria region and there are three equilibria, one of which is unstable, and the other two stable; if $\delta>0$, the state of the system is outside of the multiple equilibria region and there is a unique equilibrium, which is stable. 
Table 2 shows the estimated parameters and their corresponding standard errors for all six countries. The parameters $\lambda$ and $\sigma$ shift and scale the observed state variable. In the fit the location parameter $\lambda$ is assumed to be equal to 0 since there is a fundamental equilibrium at $y=0$. The $\mathrm{AIC}$ and $\mathrm{BIC}$ indicate the in-sample fitness of the model (Hartelman, 1997; Grasman et al., 2009) based on the log residual sum of squares, penalized for the number of model parameters. The standard errors of the transformed parameters of Cardan's discriminant $\delta$ are obtained by the functional delta method.

Table 2: Estimation results assuming constant $\alpha$ and $\beta$. The location parameter $\lambda$ was fixed at zero. The values in brackets denote standard errors.

\begin{tabular}{ccccccc}
\hline & $\mathrm{US}$ & $\mathrm{JP}$ & $\mathrm{UK}$ & $\mathrm{NL}$ & $\mathrm{SE}$ & $\mathrm{BE}$ \\
\hline$\sigma$ & $5.298 e+00$ & $3.420 e+00$ & $3.056 e+00^{*}$ & $2.982 e+00^{*}$ & $2.405 e+00$ & $2.641 e+00^{* * *}$ \\
$($ s.e. $)$ & $(2.858 e+00)$ & $(2.041 e+00)$ & $(1.261 e+00)$ & $(1.255 e+00)$ & $(1.348 e+00)$ & $(3.730 e-01)$ \\
$\alpha$ & $7.704 e-05$ & $-4.040 e-04$ & $1.781 e-03$ & $1.448 e-03$ & $2.583 e-05$ & $\begin{array}{c}3.058 e-03^{* *} \\
(9.538 e-04)\end{array}$ \\
$($ s.e. $)$ & $(5.314 e-04)$ & $(4.537 e-04)$ & $(1.370 e-03)$ & $(1.361 e-03)$ & $(9.484 e-04)$ & $(9.04)$ \\
$\beta$ & $-9.632 e-03$ & $1.354 e-02$ & $1.376 e-02$ & $1.353 e-02$ & $1.131 e-02$ & $4.813 e-02^{* * *}$ \\
$($ s.e. $)$ & $(1.795 e-02)$ & $(1.221 e-02)$ & $(1.765 e-02)$ & $(1.756 e-02)$ & $(2.489 e-02)$ & $(1.099 e-02)$ \\
$\delta$ & $3.735 e-06$ & $-5.528 e-06$ & $7.526 e-05$ & $4.673 e-05$ & $-5.773 e-06$ & $-1.934 e-04$ \\
$($ s.e. $)$ & $(1.842 e-05)$ & $(2.368 e-05)$ & $(1.070 e-04)$ & $(8.298 e-05)$ & $(3.799 e-05)$ & $(2.146 e-04)$ \\
\hline $\mathrm{AIC}$ & -983.611 & -901.021 & -715.484 & -721.421 & -592.250 & -782.153 \\
$\mathrm{BIC}$ & -971.021 & -888.431 & -702.894 & -708.831 & -580.719 & -770.191 \\
\hline
\end{tabular}

$* * *$ significant at $0.1 \%$ level, $* *$ significant at $1 \%$ level, $*$ significant at $5 \%$ level, $\cdot$ significant at $10 \%$ level.

Fig. 5 gives a visual illustration of the estimated values of the control parameters for the different countries on the projection of cusp equilibrium surface in Fig. 2. The cusp shaped shaded area is the region of the parameter space for which there are multiple equilibria. As shown in the legend, each symbol and color indicates the estimated parameters for one country. The ellipse around it in the same color corresponds to its $95 \%$ confidence region. With 95\% confidence, these regions include the points representing the actual control parameter values. As the estimation results in Table 2 show, the point estimates of $(\alpha, \beta)$ for the US, NL and UK are in the single equilibrium region, while JP, SE and BE are inside the gray multiple equilibria region. Note, however, that since the confidence areas for all countries considered contain points in the single as well as multiple equilibria region, one cannot state with $95 \%$ confidence for any of the six countries considered, that $(\alpha, \beta)$ is within either region. This is reflected by the fact that $\delta$ does not differ significantly from 
zero at the $5 \%$ significance level for any of the six countries considered (Table 2). Apart from the insignificant $\delta$-values, graphically the confidence sets for BE and US appear to be the least ambiguous, in that these nearly lie entirely inside and outside the multiple equilibrium cusp region, repectively.

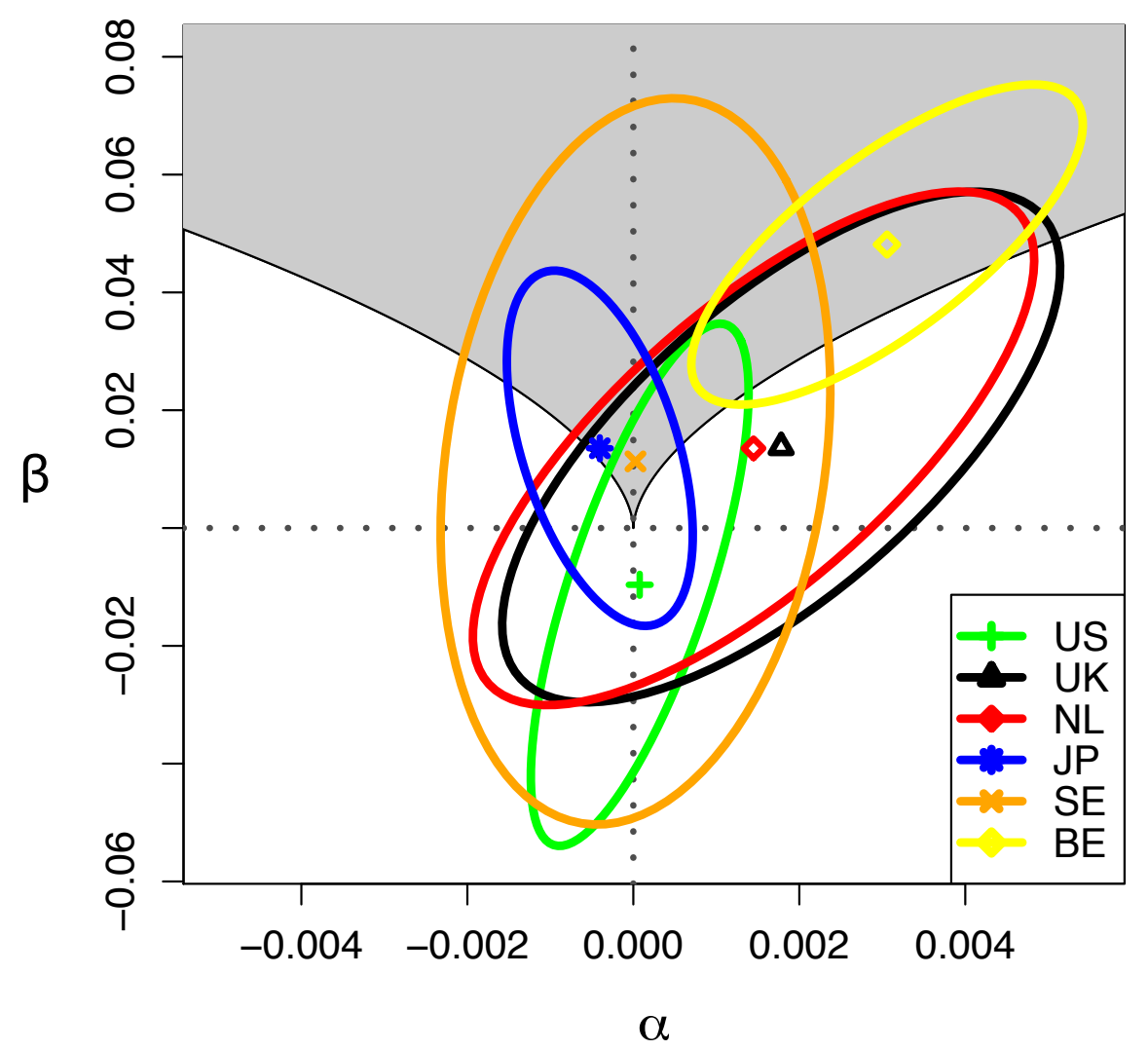

Figure 5: The estimated behavior points of different countries in different regions of the control plane with respect to constant $\alpha$ and $\beta$. The cusp shaped shaded area is the control plane. The ellipses correspond to confidence regions with confidence level of $95 \%$.

By combining Fig. 5 with the cusp equilibrium surface in Fig. 2, we can infer the most likely dynamical behavior of the housing market by observing where its state parameters are located on the cusp equilibrium surface. For instance, BE is most likely inside the multiple equilibria region where the surface predicts two stable state values instead of one. With changing control parameters, its state could then follow the path A in Fig. 2 and move close to the fold curve. A tiny perturbation on the control variables would induce it to fall 
off the cusp "cliff" and jump to a different equilibrium suddenly. The US housing market is most likely outside the dangerous multiple equilibria regime; if the control variable $\alpha$ would change, its state point would then follow a path similar to path B in Fig. 2, and the transition between equilibria would be slow and smooth without experiencing critical transitions. We could describe analogous scenarios for the other countries, albeit with larger uncertainty about whether they are in the cusp region of the parameter space.

\subsection{Interest Rate as Control Variable}

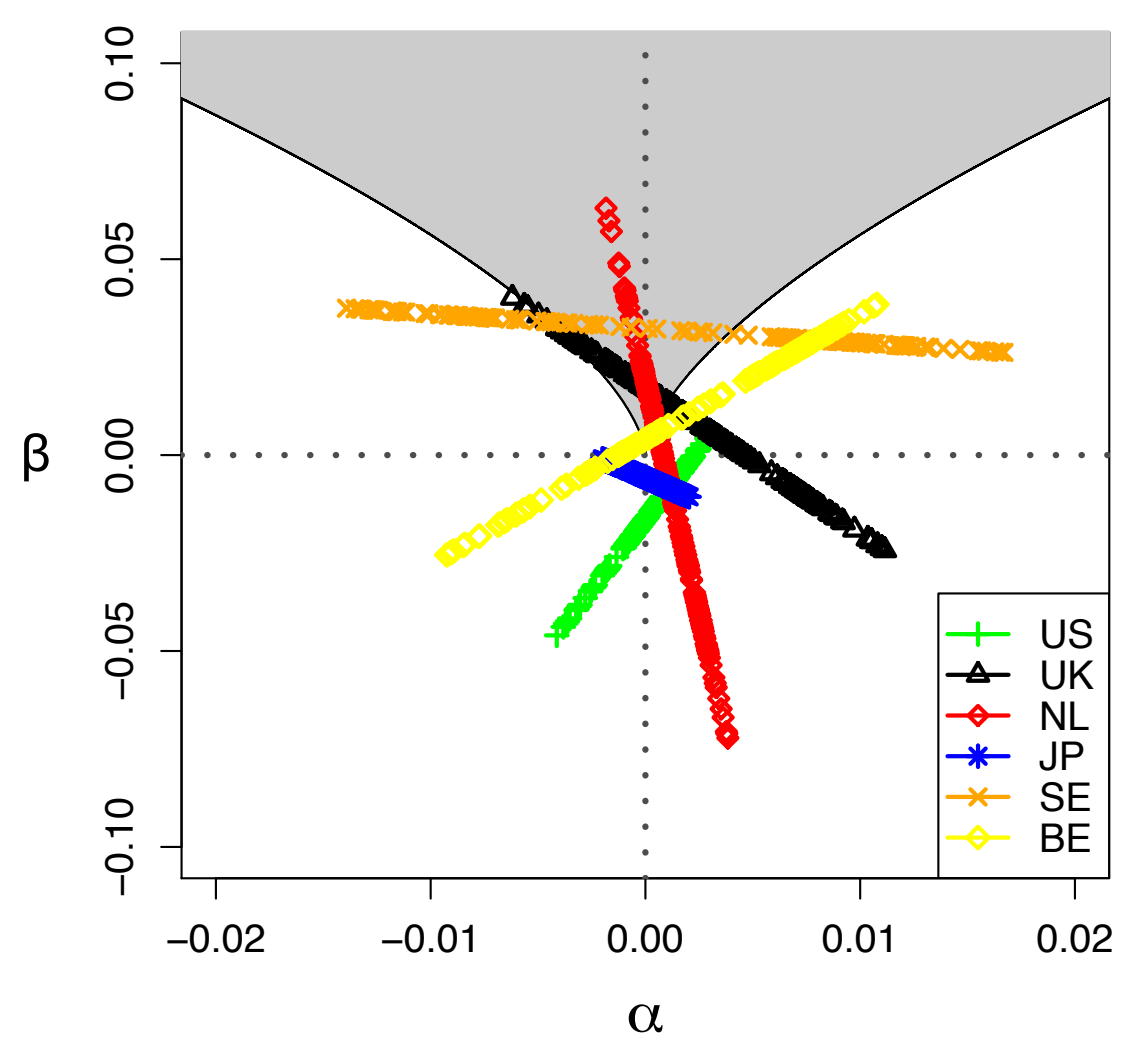

Figure 6: Estimated control parameters for different countries in different regions of the control plane. The control variable is the interest rate. The cusp-shaped shaded area is the control plane.

We next study the situation when the interest rate is used as an exogenous control variable driving the housing market. Following the estimation methods in Section 3, the control parameters $\alpha$ and $\beta$ are now defined by $\alpha=\alpha_{0}+\alpha_{1} x$ and $\beta=\beta_{0}+\beta_{1} x$, where $x$ 
Table 3: Estimated parameters $\sigma, \alpha_{0}, \alpha_{1}, \beta_{0}$ and $\beta_{1}$ when using interest rate as the control variable. The location parameter $\lambda$ was fixed at zero. The values in brackets denote standard errors.

\begin{tabular}{ccccccc}
\hline & $\mathrm{US}$ & $\mathrm{JP}$ & $\mathrm{UK}$ & $\mathrm{NL}$ & $\mathrm{SE}$ & $\mathrm{BE}$ \\
\hline$\sigma$ & $2.097 e+00$ & $4.482 e+00$ & $2.773 e+00^{* *}$ & $7.495 e+00$ & $1.485 e+00^{* * *}$ & $2.533 e+00^{* * *}$ \\
$($ s.e. $)$ & $(1.985 e+00)$ & $(4.639 e+00)$ & $(9.724 e-01)$ & $(2.141 e+01)$ & $(2.863 e-01)$ & $(3.422 e-01)$ \\
$\alpha_{0}$ & $3.989 e-03$ & $-2.279 e-03$ & $1.318 e-02^{*}$ & $4.748 e-03$ & $2.026 e-02^{* * *}$ & $1.475 e-02^{* * *}$ \\
$(s . e)$. & $(4.267 e-03)$ & $(2.498 e-03)$ & $(5.629 e-03)$ & $(1.361 e-02)$ & $(5.808 e-03)$ & $(2.678 e-03)$ \\
$\alpha_{1}$ & $-5.466 e-04$ & $4.643 e-04$ & $-1.209 e-03^{*}$ & $-5.487 e-04$ & $-2.491 e-03^{* * *}$ & $-1.737 e-03^{* * *}$ \\
$(s . e)$. & $(5.671 e-04)$ & $(4.926 e-04)$ & $(5.134 e-04)$ & $(1.556 e-03)$ & $(6.659 e-04)$ & $(3.308 e-04)$ \\
$\beta_{0}$ & $1.232 e-02$ & $-2.995 e-04$ & $-3.181 e-02$ & $-9.390 e-02^{*}$ & $2.489 e-02$ & $5.117 e-02^{*}$ \\
$($ s.e. $)$ & $(4.638 e-02)$ & $(1.572 e-02)$ & $(3.449 e-02)$ & $(4.172 e-02)$ & $(3.020 e-02)$ & $(2.049 e-02)$ \\
$\beta_{1}$ & $-3.929 e-03$ & $-1.115 e-03$ & $4.491 e-03$ & $1.308 e-02^{* *}$ & $9.192 e-04$ & $-5.549 e-03^{*}$ \\
$($ s.e. $)$ & $(6.085 e-03)$ & $(3.199 e-03)$ & $(4.052 e-03)$ & $(4.992 e-03)$ & $(2.533 e-03)$ & $(2.301 e-03)$ \\
\hline $\mathrm{AIC}$ & -988.770 & -912.718 & -732.828 & -729.114 & -630.110 & -831.070 \\
$\mathrm{BIC}$ & -969.885 & -893.832 & -713.943 & -710.229 & -612.813 & -813.128 \\
\hline
\end{tabular}

$* * *$ significant at $0.1 \%$ level, $* *$ significant at $1 \%$ level, $*$ significant at $5 \%$ level, $\cdot$ significant at $10 \%$ level.

is the interest rate. The scale parameter $\sigma$, and the coefficients $\alpha_{0}, \alpha_{1}, \beta_{0}$ and $\beta_{1}$ are the parameters to be estimated.

Table 3 shows the estimation results for the different countries. Although the parameters are not significant for a number of countries, to investigate the estimated paths of the control parameters for the different countries, these are visually displayed in Fig. 6. It can be seen that the parameters $(\alpha, \beta)$ trace different paths on the control plane. By comparing with the benchmark in Fig. 5, we shall see that the interest rate as a control variable can potentially greatly impact the stability of housing markets. For the housing markets of NL and UK, although their equilibrium points appear to be located in the stable regions under constant control parameters (Fig. 5), changes of the interest rate can induce them to move into the multiple equilibria region in certain periods (Fig. 6). On the contrary, the paths for US and JP now are outside of the multiple equilibria region.

\subsubsection{Leave-one-out cross-validation}

In order to assess whether estimated time series models have out-of-sample forecasting ability, time series are usually divided in an in-sample estimation part and a remaining hold- 
out part on which the out-of-sample forecasting ability is evaluated. However, here this is practically infeasible due to the small sample sizes (ranging from 132 to 172 observations here, depending on the country). Dividing the sample into two fairly sized subsamples would seriously affect the estimation sample size, and still only give a modest number of out-of-sample evaluations.

We therefore perform leave-one-out cross-validation (LOOCV) (Stone, 1974; Allen, 1974; Geisser, 1975) to compare the forecasting ability of our two estimated models (with and without the interest rate as control variable) with that of a simple benchmark forecast, being the naive forecast - the optimal forecast for a random walk without drift. The idea of LOOCV is to leave out the observations one by one from the sample, re-estimate the model, and forecast the observation that was left out of the sample. In this way one can obtain an out-of-sample forecast for each observation, without severely affecting the estimation sample size.

Table 4: LOOCV results (mean squared prediction errors). The smallest values per country are indicated by bold face print.

\begin{tabular}{ccccccc}
\hline MSPE $\times 10^{4}$ & US & JP & UK & NL & SE & BE \\
\hline naive forecast & $\mathbf{1 . 8 5 8}$ & 3.01 & 8.868 & 8.582 & 6.344 & 3.116 \\
cusp, no control & 1.914 & 3.10 & 9.148 & 8.876 & 6.534 & 2.825 \\
cusp, interest rate & 1.905 & $\mathbf{2 . 9 1}$ & $\mathbf{8 . 2 2 5}$ & $\mathbf{8 . 5 2 9}$ & $\mathbf{4 . 8 5 0}$ & $\mathbf{2 . 0 4 2}$ \\
\hline
\end{tabular}

Mean squared errors obtained with LOOCV for the naive forecast and the stochastic cusp model without and with interest rate as a control variable.

The LOOCV mean squared prediction errors (MSPEs) are reported in Table 4, which for comparison also contains results for the naive forecast $\hat{y}_{t+1}=y_{t}$, i.e. the last observed value. The results indicate that for all countries except US the out-of-sample forecasting ability of our model without the interest rate as a control variable performs worse than the naive benchmark, but the model with the interest rate as control variable outperforms both other models. The US is an exception for which the naive forecast gives the smallest MSPE. The addition of the control variable interest rate to the cusp model in all cases leads to smaller MSPEs. Upon adding more control variables the forecasting ability of the cusp model might increase further; it is beyond the scope of the present paper to investigate this. 


\subsubsection{Equilibria}
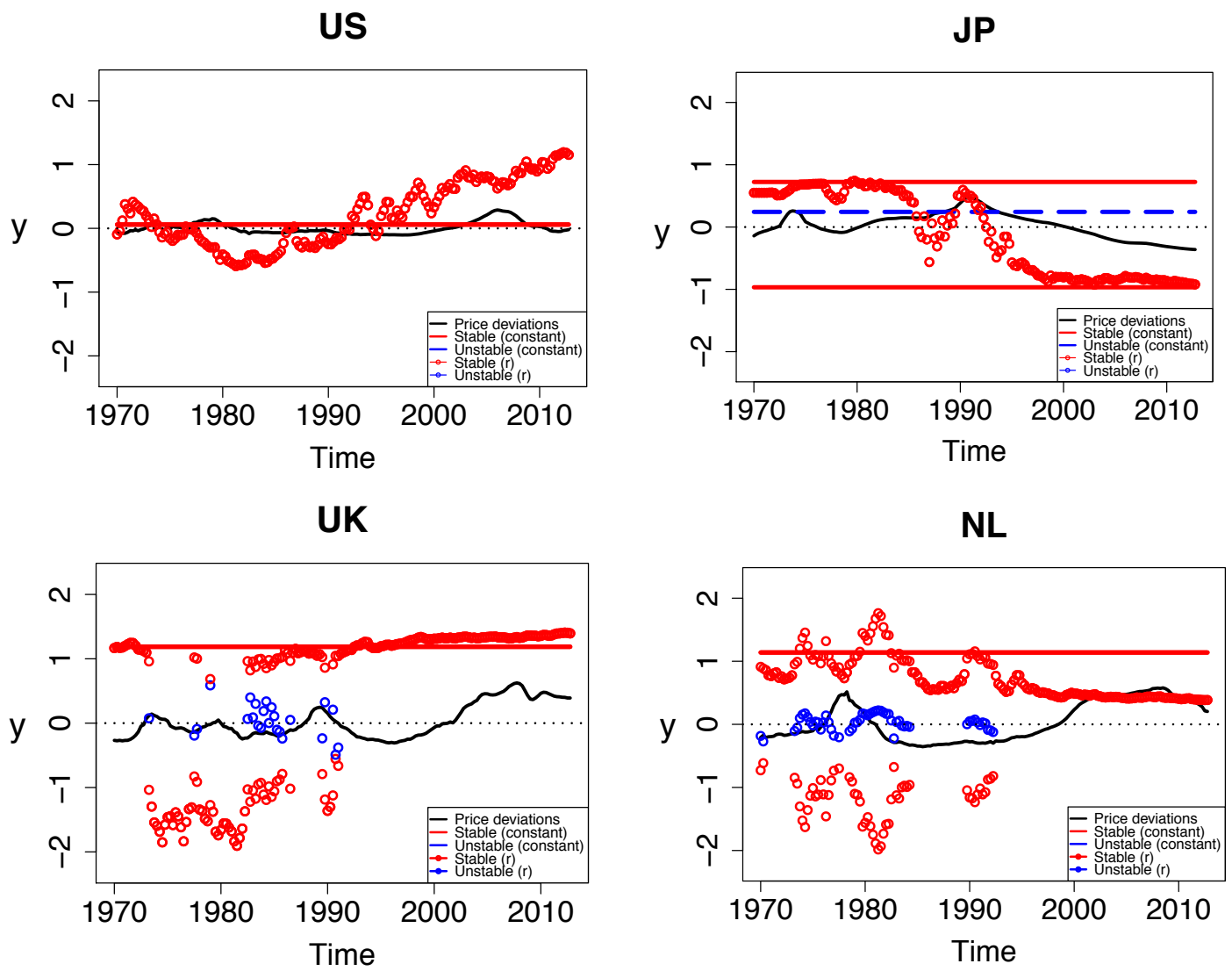

SE
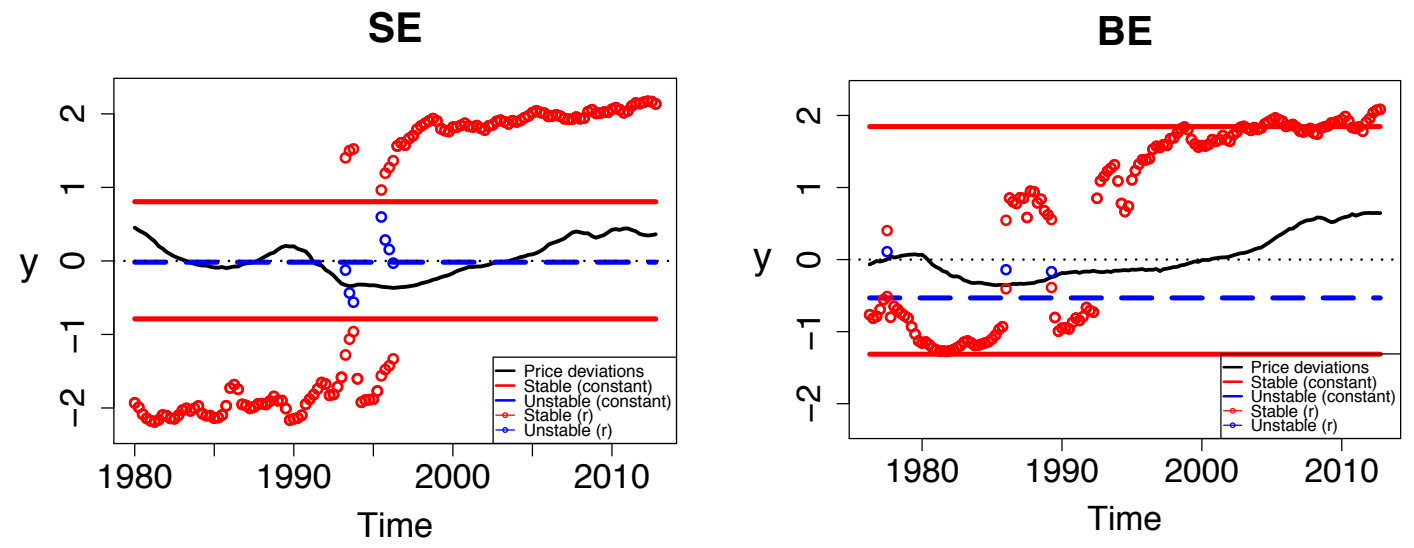

Figure 7: Time series of housing price deviations and predicted equilibria in different countries. The black line represents the time series of housing price deviations. The scatter plots indicate estimated equilibria when the interest rate is used as control variable. The red and blue lines indicate the estimated equilibria for constant parameters. 
Fig. 7 shows the model-implied equilibrium values as a function of time and the time series of housing price deviations from fundamentals for the different countries. It allows us to investigate the equilibria of the system across time, and also provides information on how the state of the system transits from one equilibrium to another.

The black line in Fig. 7 represents the time series of housing price deviations from fundamentals. The black dotted line indicates the baseline 0. All six countries exhibit long-lasting periods of fluctuations of price deviations around 0 . It can be observed that housing prices have been increasing rapidly since the mid-1990s and have peaked around 2008 in the US and NL. After that they have dropped considerably for those countries. In JP housing prices peaked earlier, around 1990, and subsequently declined to levels below the baseline 0. The UK, SE and BE housing markets exhibited peaks around 1990 and 2008, after which housing prices dropped significantly.

The red and blue horizontal lines indicate the estimated equilibria when control parameters are constant (Section 5.1), while the scatter plots show the equilibria with interest rate as control variables. The red lines or scatters represent stable equilibria on the upper or bottom sheet of cusp equilibrium surface, while the blue lines or scatters imply unstable equilibria on the middle sheet of cusp equilibrium surface.

Some of the equilibria do not align with the position of the actual data in the plots. A possible explanation is that our estimates were based on optimizing one-step-ahead price forecasts, the objective function of which is rather insensitive to the actual long-run equilibrium. From the trends in the price in the direction of the time-varying equilibria, it can be observed that the cusp model forecasts the direction of change of the equilibrium in real housing data well. For instance, in the case of SE around mid-1990s, the equilibrium presented a transition from a low price equilibrium to a high price equilibrium. Around the same time, the corresponding housing price data experienced a change from a decline towards the lower equilibrium into a gradual increase towards the upper equilibrium.

\subsubsection{Critical Transitions}

Fig. 8 displays time series of price deviations, interest rates and the cube root of Cardan's discriminant $\delta$ in different countries. Under the monitoring of monetary policy, interest rates in all countries exhibited long lasting fluctuations regarding different economic situations. In general, for JP, SE and NL, the interest rates were declining in the observed period. For the US, UK and BE, interest rates peaked around 1980, followed by persistent 

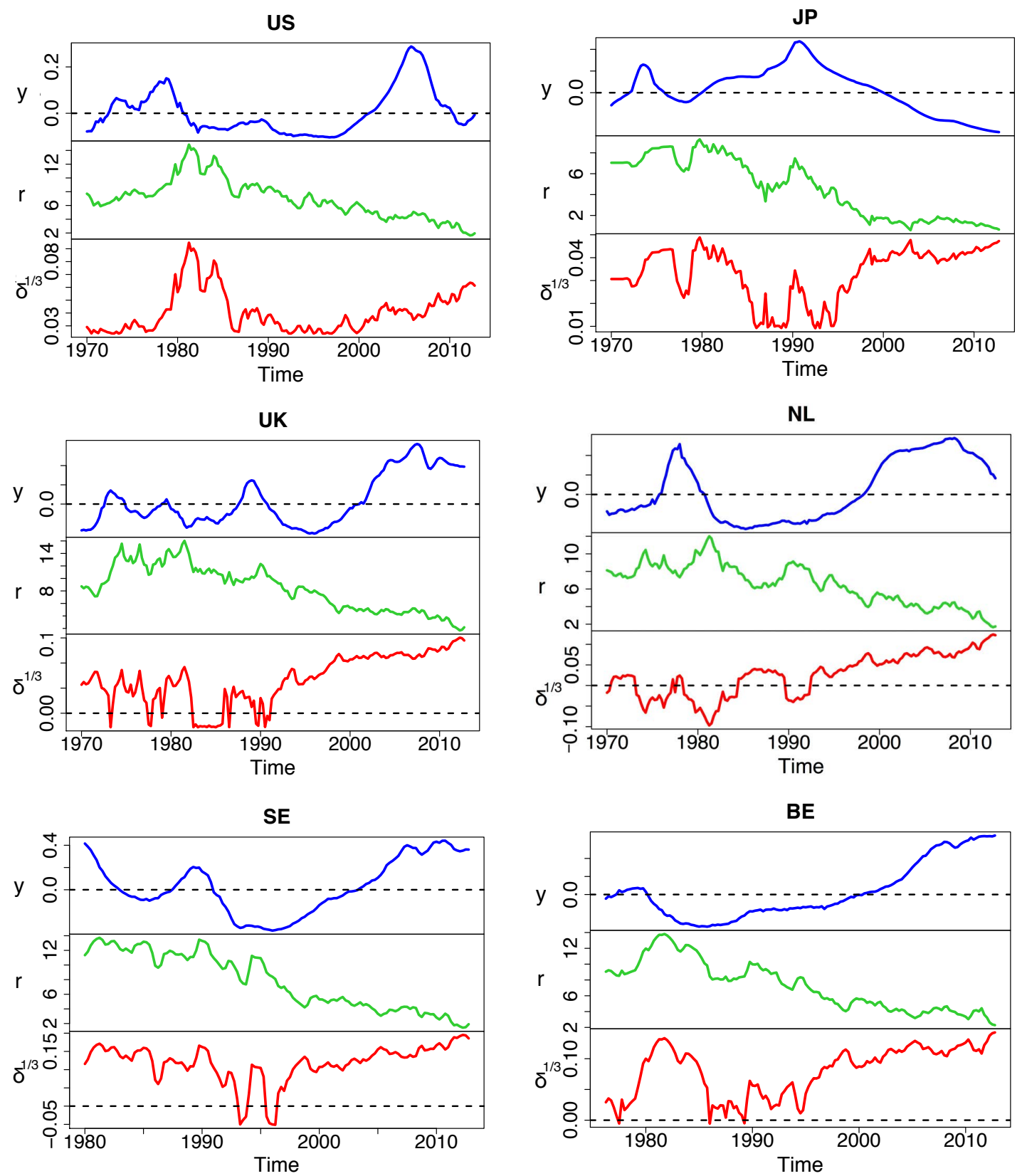

Figure 8: Time series of state variable (top panels), interest rate (middle panels) and cube root of Cardan's discriminant $\delta$ (bottom panels) in different countries. The control variable is the interest rate. The dashed line indicates the baseline of 0 .

declines.

Because $\delta$ has a very sharply peaked distribution near zero, being based on second and third powers of $\alpha$ and $\beta$, transformation to its cube root allows us to visually catch more 
details on its fluctuations around 0 . We are particularly interested in the corresponding price dynamics when $\delta$ crosses the 0 baseline from negative to positive, which implies that the state of system moves from the multiple equilibria region to the stable region. This can be observed during most of the housing price bubble and burst cycles in the UK, NL, $\mathrm{SE}$ and BE. When crossing the bifurcation boundary from inside the multiple equilibria region to outside, a critical transition may occur, driven by the interest rate, where the system's state 'falls' off the cusp curve and transitions from one equilibrium to another.

Large fluctuations in the price time series can be either shock driven near a single stable equilibrium or a critical transition. To help interpret observed price changes a direct comparison of the relative price deviation $y$ with the control variable $r$ is useful. Fig. 9 illustrates how the time series of the control variable - the interest rate, corresponds with the various stability conditions in the housing market historically. The gray 'bifurcation band' indicates the range of interest rate values with respect to $\delta<0$, corresponding to the multiple equilibria region with three equilibria - two stable and one unstable. A critical transition happens when the state of system crosses the band entirely, and this graph helps identifying when this happened.

By observing the relationship between interest rates and the multiple equilibria region, displayed across time in Fig. 9, we are able to study the underlying mechanisms of fluctuations in housing markets. For instance, in the example of SE, the state of the housing system has fallen into the multiple equilibria region twice between 1990 and 2000. This corresponds with the bottom of the downturn of the housing price index. After falling into the multiple equilibria region for the first time, the state of the system quickly came out of it and went back to the previous stable equilibrium due to a rise of the interest rate. A year later the interest rate dropped again and the system was brought down to the multiple equilibria region for the second time. This time, it did not end up in the previous equilibrium but transitioned to a new stable equilibrium after moving out of the bifurcation band on the other side. This transition induced the retrieval of the housing market. Since then, the SE housing price has been continuously increasing. The UK and BE show similar bifurcation bands in their interest rates and exhibited similar systematic fluctuations as SE. They had experienced several critical transitions between two equilibria before the mid-1990s, which were consistent with the fluctuations in real housing price. After that, the UK came back to its previous stable equilibrium. This behavior corresponded with the recovery of housing market in these two countries. In particular, the housing 


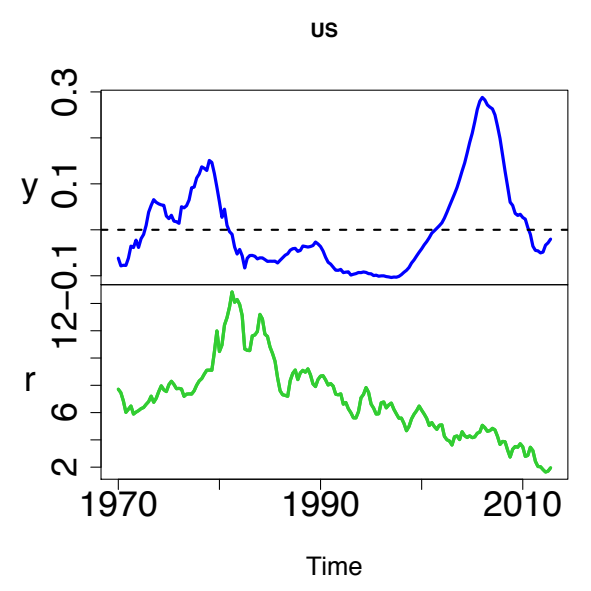

UK
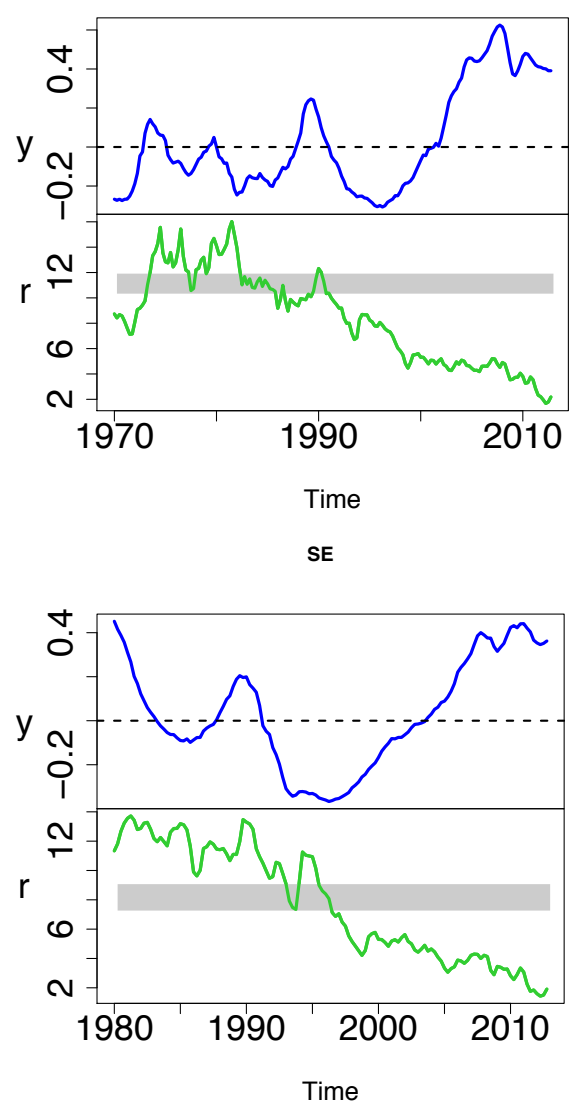

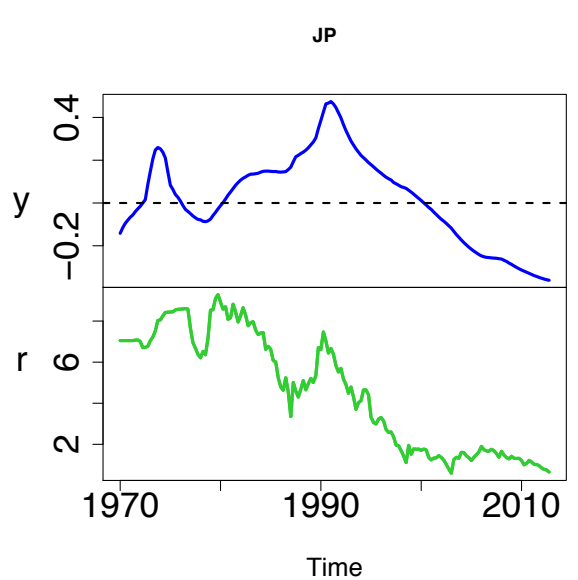

NL

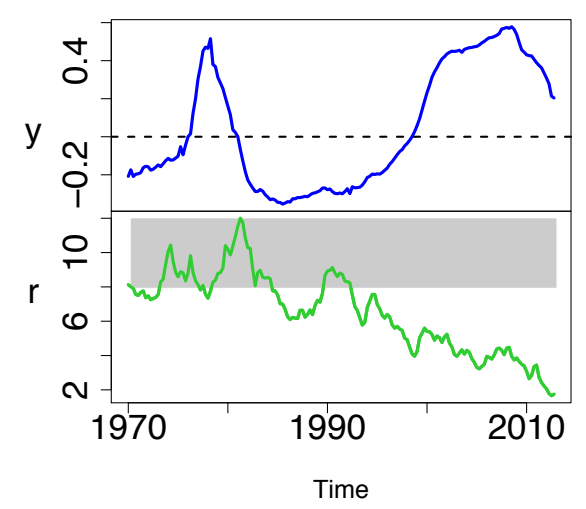

BE

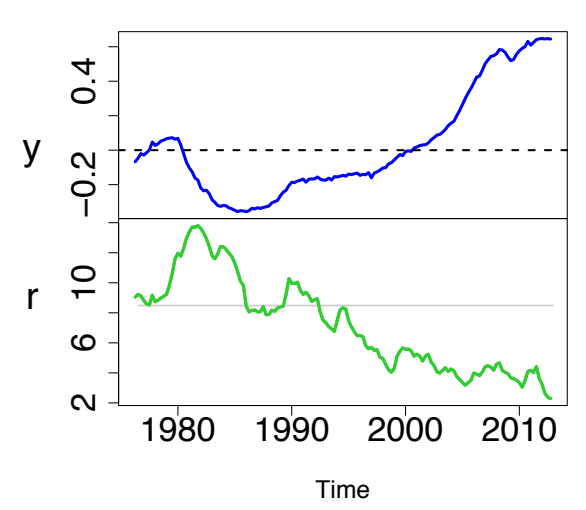

Figure 9: Time series of housing price deviations, and interest rate with gray bifurcation band indicating the region of $r$-values where there are three equlibria.

system of NL has the widest bifurcation band of all. It has been in the multiple equilibria region for about a decade between 1973 and 1984, while the corresponding housing price 
was experiencing a bubble and burst cycle. Nevertheless, its equilibrium never went fully across the bifurcation band. For the US and JP, there is no bifurcation band. According to our analysis, their housing systems remained in the single equilibrium regime.

To analyze the impact of interest rate on the equilibrium of housing system further, Fig. 10 presents the bifurcations showing the predicted equilibria as a function of the interest rate $r$ for the different countries. The red dots represent the stable equilibria on the upper or bottom sheet of cusp equilibrium surface, and the blue dots represent the unstable equilibria on the middle cusp sheet. This reveals the underlying bifurcations in different housing systems. For the UK, SE and BE, the observed bifurcations are two connected saddle-node bifurcations. With interest rate as a control parameter, the cusp model exhibits three equilibria for a certain range of interest rate, and a single equilibrium outside this range. The bifurcation scenario for the example of NL is different, in that it shows only one bifurcation. For small values of the interest rate there is one quilibrium, and for larger interest rates than about 7.5\% there are three equilibria. Increasing the interest rate further does not lead to a single equilibrium again, as it does for the UK, SE and $\mathrm{BE}$. This is related to the fact that the estimated line with possible $(\alpha, \beta)$-values for the Netherlands only crosses the bifurcation border once in the $(\alpha, \beta)$ plane (see Fig. 6 ), while those for UK, SE and BE cross it twice, entering the cusp-shaped multiple equilibria region on the negative $\alpha$ side and exiting it on the positive $\alpha$ side. For the US and JP, no bifurcations occurred during the analyzed period.

\subsubsection{Policy Implications}

Historically housing busts have often been followed by financial crises, which is why housing booms raise great concerns regarding the instability of housing prices among policy makers. How can a policy maker stabilize the housing price and prevent market instabilities? As an essential factor in the monetary policy, the interest rate has been pointed out to have great influence on the (in)stability of housing markets (Bernanke and Gertler, 1995; Shiller, 2006; Muellbauer and Murphy, 2008; Taylor, 2007, 2009; Crowe et al., 2013; Shi et al., 2014). Our study once again sheds light on the importance of interest rates. Moreover, we reveal the underlying link between interest rate and systematic fluctuations in housing markets.

The dynamics of the housing system shows cusp catastrophe behavior with interest rate as the control parameter. It exhibits critical transitions between multiple equilibrium states as a result of the changes of interest rate. It is dangerous when the system is in the 

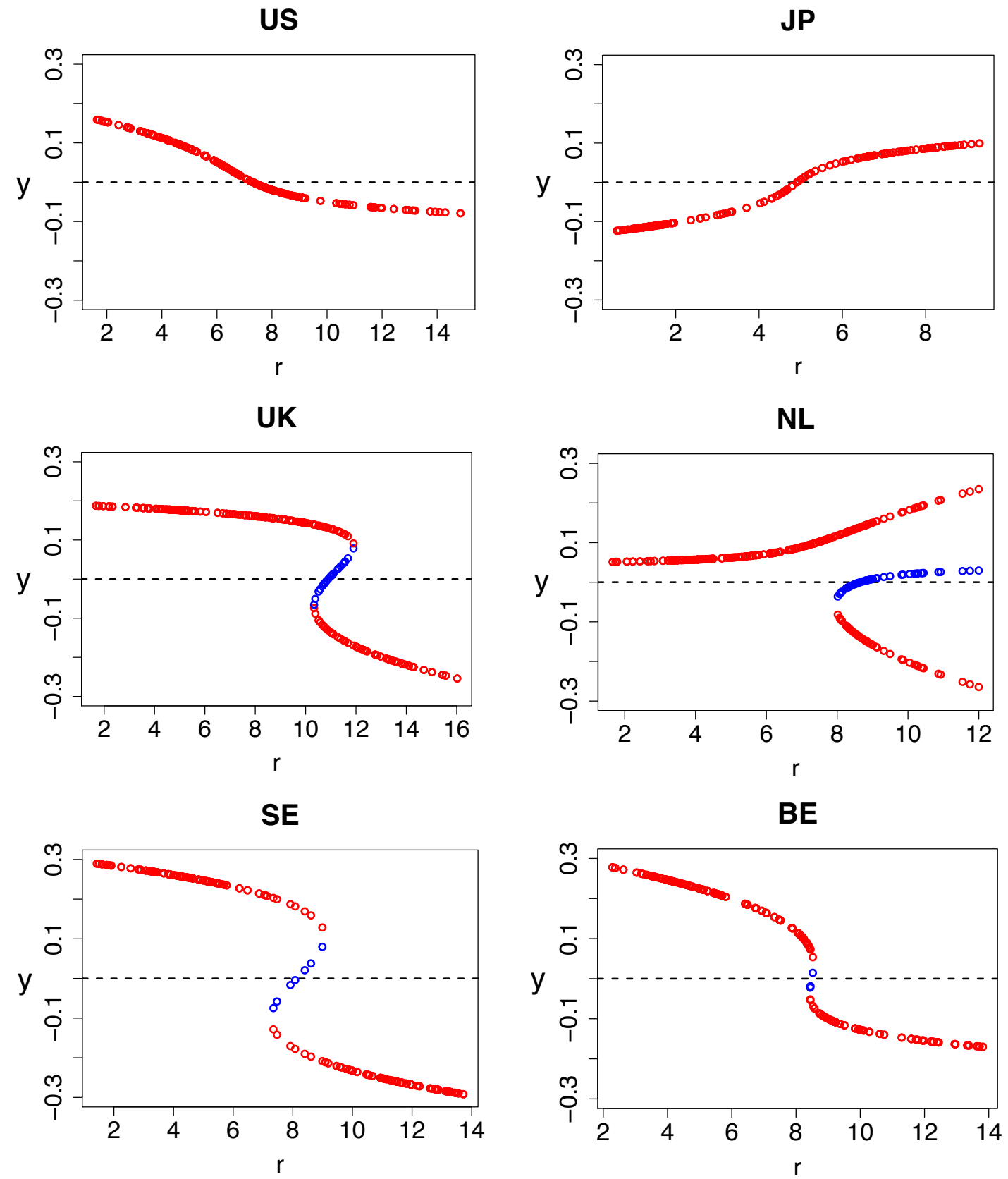

Figure 10: Bifurcations showing the predicted equilibria as a function of the interest rate $r$ for the different countries. Red scatter represents the stable equilibrium (up or bottom sheet). Blue scatter represents the unstable equilibrium (middle sheet)

multiple equilibria region, and gets too close to the cusp curve. Even a small perturbation could induce a critical transition there. This scenario can be used to explain the majority 
of housing booms and busts in the data, such as in UK 1978, 1980,1990, NL 1978, BE 1987,1990, and the depression of SE after 1990.

A general lesson for policy makers to be drawn from these examples is that the cusp catastrophe model may yield important insights on policies that can cause global instability in economics. As we argued in our analysis, policy makers should monitor the instability of economic systems, and be alert when the system approaches a bifurcation, in particular be aware of critical transitions which could lead to significant market bubbles or sudden market collapse. The examples in this paper suggest that interest rate policy plays an important role in maintaining the stability of the economic system. By performing an appropriate interest rate policy, policy makers are able to prevent endogenous market crashes. There is no empirical evidence to show whether a high or a low interest rate should be beneficial to the economy. Taking the SE housing market as an example, as seen in Fig. 10, if we move the interest rate upward to values that are too high, i.e. above $9 \%$, the market might collapse. However, when the interest rate is lowered below 7\%, bubbles may arise. Our method gives us an overall picture about the multiple equilibria of the system and how the equilibria change with interest rate policy. It could provide policy makers with a reasonable guide to conduct a proper interest rate policy that keeps the economy in a healthy state. It could also help to deal with markets with bubbles and to establish post-crisis policy on the recession following a housing market collapse.

\section{Concluding Remarks}

In this paper we investigated whether instability of housing markets can be explained and predicted by catastrophe theory by fitting a stochastic cusp catastrophe model to empirical housing market data. Using housing price deviations and quarterly data on long term government interest rates, we estimated the model for six different countries: US, UK, NL, JP, SE and BE.

Two estimation approaches are discussed - Cobb's method and Euler discretization. The analysis shows that Cobb's Method is more suitable for cross-sectional data, or for a system for which the state variables change fast compared to the sampling period and the control parameters. It performs well when modeling the overall stationary density of state variables. However, when it comes to one-step-ahead forecasting, Euler discretization gave better predictions. Because moreover our study was based on time series data and 
one of our objective was to study the forecasting ability of cusp catastrophe model, Euler discretization was employed in the later sections.

We found that the dynamics of the housing market can be explained by cusp catastrophe behavior. Under constant control parameters, the housing systems of US, UK and NL appear to be in 'normal' single equilibrium regimes and those of SE, JP and BE in multiple equilibria regimes. Nevertheless, when using interest rate as control variable, the interest rate changes the stability of the systems; those systems' equilibria vary with interest rate. The predicted equilibria give us a country-dependent global picture on the changes of equilibria as a function of the interest rate. Time series of Cardan's discriminant $\delta$ link the changes of system equilibria and the booms and busts cycles in empirical data. Moreover, by observing the relationship between interest rates and bifurcation bands, we are able to study the underlying mechanisms of fluctuations in housing markets. A critical transition can be distinguished when the interest rate crosses its bifurcation band entirely, that is, enters it from one side and exits it on the other.

Our results yield important insights into policies that monitor the instability in housing markets. A change of the main control parameter, the interest rate, may move the system closer to the edge of the multiple equilibria region, making critical transitions more likely to occur. As a control variable, the interest rate plays an important role in keeping the stability of economic system. Policy makers should prevent the economic system from moving into the multiple equilibria regions, or, when already there, from getting too close to the cusp curve that may induce critical transitions. Cusp catastrophe theory could provide policy makers with a reasonable guidebook on interest rate policy.

\section{References}

Aerts, D., Czachor, M., Gabora, L., Kuna, M., Posiewnik, A., Pykacz, J. and Syty, M. (2003). Quantum morphogenesis: A variation on Thom's catastrophe theory. Physical Review E, 67, number 5, 051926.

Alexander, R. A., Herbert, G. R., DeShon, R. P. and Hanges, P. J. (1992). An examination of least-squares regression modeling of catastrophe theory. Psychological Bulletin, 111, number 2, 366-374. 
Allen, D. M. (1974). The relationship between variable selection and data augmentation and a method for prediction. Technometrics, 16, 125-127.

Anufriev, M. and Branch, W.A. (2009). Introduction to special issue on complexity in economics and finance. Journal of Economic Dynamics and Control, 33, 1019-1022.

Barunik, J. and Kukacka, J. (2015). Realizing stock market crashes: Stochastic cusp catastrophe model of returns under the time-varying volatility. Quantitative Finance, 15, number 6, 959-973.

Barunik, J. and Vosvrda, M. (2009). Can a stochastic cusp catastrophe model explain stock market crashes? Journal of Economic Dynamics and Control, 33, number 10, $1824-1836$.

Bernanke, B. S. and Gertler, M. (1995). Inside the black box: The credit channel of monetary policy transmission. The Journal of Economic Perspectives, 9, number 4, $27-48$.

Bolt, W., Demertzis, M., Diks, C., Hommes, C.H. and van der Leij, M. (2014). Identifying booms and busts in house prices under heterogeneous expectations. De Nederlandsche Bank Working paper, 450.

Boswijk, H P., Hommes, C.H. and Manzan, S. (2007). Behavioral heterogeneity in stock prices. Journal of Economic Dynamics and Control, 31, number 6, 1938-1970.

Brillinger, D. R. (2007). Learning a potential function from a trajectory. IEEE Signal Processing Letters, 14, number 11, 867-870.

Brock, W.A. and Hommes, C.H. (1997). A rational route to randomness. Econometrica, 65, number 5, 1059-1095.

Brock, W.A. and Hommes, C.H. (1998). Heterogeneous beliefs and routes to chaos in a simple asset pricing model. Journal of Economic Dynamics and Control, 22, number 8-9, 1235-1274.

Case, K.E. and Shiller, R.J. (2003). Is there a bubble in the housing market? Brookings Papers on Economic Activity, 2003, 299-342. 
Cobb, L. (1978). Stochastic catastrophe models and multimodal distributions. Behavioral Science, 23, number 4, 360-374.

Cobb, L. (1981a). Estimation theory for the cusp catastrophe model. Proceedings of the Section on Survey Research Mehods, 26, 772-776.

Cobb, L. (1981b). Parameter estimation for the cusp catastrophe model. Behavioral Science, 26, number 1, 75-78.

Cobb, L., Koppstein, P. and Chen, N. H. (1983). Estimation and moment recursion relations for multimodal distributions of the exponential family. Journal of the American Statistical Association, 78, number 381, 124-130.

Cobb, L. and Watson, B. (1980). Statistical catastrophe theory: An overview. Mathematical Modelling, 1, number 4, 311-317.

Crowe, C., Dell'Ariccia, G., Igan, D. and Rabanal, P. (2013). How to deal with real estate booms: Lessons from country experiences. Journal of Financial Stability, 9, number 3, 300-319.

Davis, M.A. and Heathcote, J. (2005). Housing and the business cycle. International Economic Review, 46, 751-784.

Dieci, R. and Westerhoff, F. (2012). A simple model of a speculative housing market. Journal of Evolutionary Economics, 22, number 2, 303-329.

Dieci, R. and Westerhoff, F. (2013). Modeling house price dynamics with heterogeneous speculators. In Global Analysis of Dynamic Models in Economics and Finance, pp. 35-61. Springer.

Dieci, R. and Westerhoff, F. (2015). Heterogeneous expectations, boom-bust housing cycles, and supply conditions: a nonlinear dynamics approach. Technical Report. BERG. Working paper 99 .

Florens-Zmirou, D. (1989). Approximate discrete-time schemes for statistics of diffusion processes. Statistics, 20, 547-557.

Geisser, S. (1975). The predictive sample reuse method with applications. Journal of the American Statistical Association, 70, 320-328. 
Gilmore, R. (1993). Catastrophe theory for scientists and engineers. Dover, New York, US.

Grasman, R. P., van der Maas, H. L. J. and Wagenmakers, E. (2009). Fitting the cusp catastrophe in R: A cusp-package primer. Journal of Statistical Software, 32, number 8, 1-28.

Guastello, S. J. (1988). Catastrophe modeling of the accident process: Organizational subunit size. Psychological Bulletin, 103, number 2, 246-255.

Hartelman, P.A.I. (1997). Stochastic catastrophe theory. Ph.D. Thesis. University of Amsterdam.

Hartelman, P.A.I., van der Maas, H. L. J. and Molenaar, P. C. M. (1998). Detecting and modelling developmental transitions. British Journal of Developmental Psychology, 16, number $1,97-122$.

Hommes, C. H. (2013). Behavioral Rationality and Heterogeneous Expectations in Complex Economic Systems. Cambridge University Press.

Kouwenberg, R. and Zwinkels, R. (2015). Endogenous price bubbles in a multi-agent system of the housing market. PLOS ONE, 10, 1-10; article number e0129070.

Lange, R., Oliva, T. A. and McDade, S. R. (2000). An algorithm for estimating multivariate catastrophe models: GEMCAT II. Studies in Nonlinear Dynamics 86 Econometrics, 4, number 3, 137-168.

Muellbauer, J. and Murphy, A. (2008). Housing markets and the economy: The assessment. Oxford Review of Economic Policy, 24, number 1, 1-33.

Oliva, T. A., Desarbo, W. S., Day, D. L. and Jedidi, K. (1987). GEMCAT: A general multivariate methodology for estimating catastrophe models. Behavioral Science, 32, number 2, 121-137.

Pavliotis, G.A. (2014). Stochastic Processes and Applications. Texts in Applied Mathematics, volume 60. Springer, NY.

Poston, T. and Stewart, I. (2012). Catastrophe theory and its applications, 1 edn. Dover, New York, US. 
Reinhart, C.M. and Rogoff, K.S. (2009). This time is different: Eight centuries of financial folly. Princeton Univ Press, Princeton, US.

Shi, S., Jou, J. and Tripe, D. (2014). Can interest rates really control house prices? Effectiveness and implications for macroprudential policy. Journal of Banking \& Finance, 47, $15-28$.

Shiller, R. J. (2006). Long-term perspectives on the current boom in home prices. The Economists' Voice, 3, number 4, 1-11.

Shiller, R. J. (2007). Understanding recent trends in house prices and home ownership. Technical Report. NBER. Working Paper w13553.

Shiller, R. J. (2008). Historic turning points in real estate. Eastern Economic Journal, 34, number 1, 1-13.

Shiller, R. J. (2012). The subprime solution: How today's global financial crisis happened, and what to do about it. Princeton University Press, Princeton, US.

Shiller, R. J. (2015). Irrational exuberance. Princeton University Press, Princeton, US.

Stone, M. (1974). Cross-validatory choice and assessment of statistical predictions. Journal of the Royal Statistical Society Series B, 36, 111-147.

Tamaki, T., Torii, T. and Maeda, K. (2003). Stability analysis of black holes via a catastrophe theory and black hole thermodynamics in generalized theories of gravity. Physical Review D, 68, number 2, 024028.

Taylor, J. B. (2007). Housing and monetary policy. Proceedings - Economic Policy Symposium - Jackson Hole, Federal Reserve Bank of Kansas City, 463-476.

Taylor, J. B. (2009). The financial crisis and the policy responses: An empirical analysis of what went wrong. Critical Review: A Journal of Politics and Society, 21, 341-364.

Thom, R. (1972). Structural stability and morphogenesis. Benjamin, New York, US.

Van der Maas, H. L., Kolstein, R. and Van Der Pligt, J. (2003). Sudden transitions in attitudes. Sociological Methods \& Research, 32, number 2, 125-152. 
Van der Maas, H. L. and Molenaar, P. C. (1992). Stagewise cognitive development: An application of catastrophe theory. Psychological review, 99, number 3, 395-417.

Wagenmakers, E., Molenaar, P., Grasman, R. P., Hartelman, P. A. and van der Maas, H. (2005). Transformation invariant stochastic catastrophe theory. Physica D: Nonlinear Phenomena, 211, number 3, 263-276.

Wilson, A. (1981). Catastrophe theory and bifurcation: Applications to urban and regional systems. Croom Helm, London; Berkeley University of California Press, Berkeley, US.

Yoshida, N. (1992). Estimation for diffusion processes from discrete observation. Journal of Multivariate Analysis, 41, 220-242.

Zeeman, E. C. (1974). On the unstable behaviour of stock exchanges. Journal of Mathematical Economics, 1, number 1, 39-49.

Zeeman, E. C. (1977). Catastrophe theory: Selected papers, 1972-1977. Addison-Wesley, London, Amsterdam, Ontario, Sydney, Tokyo. 


\section{A Cobb's Method v.s. Euler Discretization}

\section{A.1 Parameterizations}

Suppose that the deterministic part of the (canonical) variable $z_{t}$ is governed by the potential function

$$
V(z ; \alpha, \beta)=-\alpha z-\frac{1}{2} \beta z^{2}+\frac{1}{4} z^{4},
$$

and that there is a driving noise term with variance $\sigma_{z}^{2}$ per time unit, i.e.

$$
\mathrm{d} z_{t}=-\left.\frac{\partial V(z ; \alpha, \beta)}{\partial z}\right|_{z=z_{t}} \mathrm{~d} t+\sigma_{z} \mathrm{~d} W_{t} .
$$

The stationary density of $z$ then is proportional to (Gibbs distribution)

$$
f_{Z}(z) \propto \exp \left[-\frac{2 V(z)}{\sigma_{z}^{2}}\right]=\exp \left[\frac{\alpha z+\frac{1}{2} \beta z^{2}-\frac{1}{4} z^{4}}{\sigma_{z}^{2} / 2}\right],
$$

see e.g. Pavliotis (2014), pp. 109-110.

If $y=\lambda+\sigma z$ is a scaled and/or translated variable, then $z=(y-\lambda) / \sigma$, and the density of $y$ is proportional to

$$
f_{Y}(y) \propto \exp \left[\frac{\alpha\left(\frac{y-\lambda}{\sigma}\right)+\frac{1}{2} \beta\left(\frac{y-\lambda}{\sigma}\right)^{2}-\frac{1}{4}\left(\frac{y-\lambda}{\sigma}\right)^{4}}{\sigma_{z}^{2} / 2}\right] .
$$

Cobb's approach as implemented in the 'cusp' $\mathbf{R}$ package The stationary density as it is fitted by the 'cusp' R package implemented by Grasman et al. (2009) has the form

$$
f_{Y}(y)=\psi \exp \left[\tilde{\alpha}\left(\frac{y-\lambda}{c}\right)+\frac{1}{2} \tilde{\beta}\left(\frac{y-\lambda}{c}\right)^{2}-\frac{1}{4}\left(\frac{y-\lambda}{c}\right)^{4}\right] .
$$

Comparing the coefficients of the fourth powers in Eqs (16) and (17), we see that these coincide only if $c=\sigma\left(\frac{\sigma_{z}^{2}}{2}\right)^{\frac{1}{4}}$. It can be readily checked that this implies $\tilde{\alpha}=\left(\frac{\sigma_{z}^{2}}{2}\right)^{-\frac{3}{4}} \alpha$ and $\tilde{\beta}=\left(\frac{\sigma_{z}^{2}}{2}\right)^{-\frac{1}{2}} \beta$.

Euler discretization In terms of $y_{t}=\lambda+\sigma z_{t}$ the SDE is given by

$$
\frac{1}{r} \mathrm{~d} y_{t}=-\left.\frac{\partial V(z ; \alpha, \beta)}{\partial z}\right|_{z=\frac{y_{t}-\lambda}{\sigma}} \mathrm{d} t+\sigma_{z} \mathrm{~d} W_{t} .
$$


Euler discretization gives

$$
y_{t+\Delta t} \approx y_{t}+\left(\alpha+\beta\left(\frac{y_{t}-\lambda}{\sigma}\right)-\left(\frac{y_{t}-\lambda}{\sigma}\right)^{3}\right) r \Delta t+\sigma \sigma_{z} \sqrt{\Delta t} \varepsilon_{t+\Delta t},
$$

where $\varepsilon_{t+\Delta t} \sim N(0,1)$. Fixing $\Delta t$ to the value 1 , as we do, our estimation method based on nonlinear least squares regression fitting the r.h.s. to the observed values on the l.h.s. provides direct estimates of $\alpha, \beta, \lambda$ and $\sigma$.

Relations between estimated parameters Although CUSPfit and Euler discretization are based on the same underlying SDE, the parameters estimated by the two methods are not directly comparable. While Euler discretization directly estimates $\alpha$ and $\beta$ as they appear in the SDE, the cusp package estimates $\tilde{\alpha}$ and $\tilde{\beta}$, which are scaled versions of $\alpha$ and $\beta$. Note, however, that the sign of $\delta$ is invariant under this transformation of scale, since $\tilde{\delta} \equiv 27 \tilde{\alpha}^{2}-4 \tilde{\beta}^{2}=27\left(\frac{\sigma_{z}^{2}}{2}\right)^{-\frac{3}{2}} \alpha^{2}-4\left(\frac{\sigma_{z}^{2}}{2}\right)^{-\frac{3}{2}} \beta^{3}=\left(\frac{\sigma_{z}^{2}}{2}\right)^{-\frac{3}{2}} \delta$.

\section{A.2 Residuals}

This section provides graphs of the residuals for Cobb's approach (Fig. 11) and Euler discretization (Fig. 12)
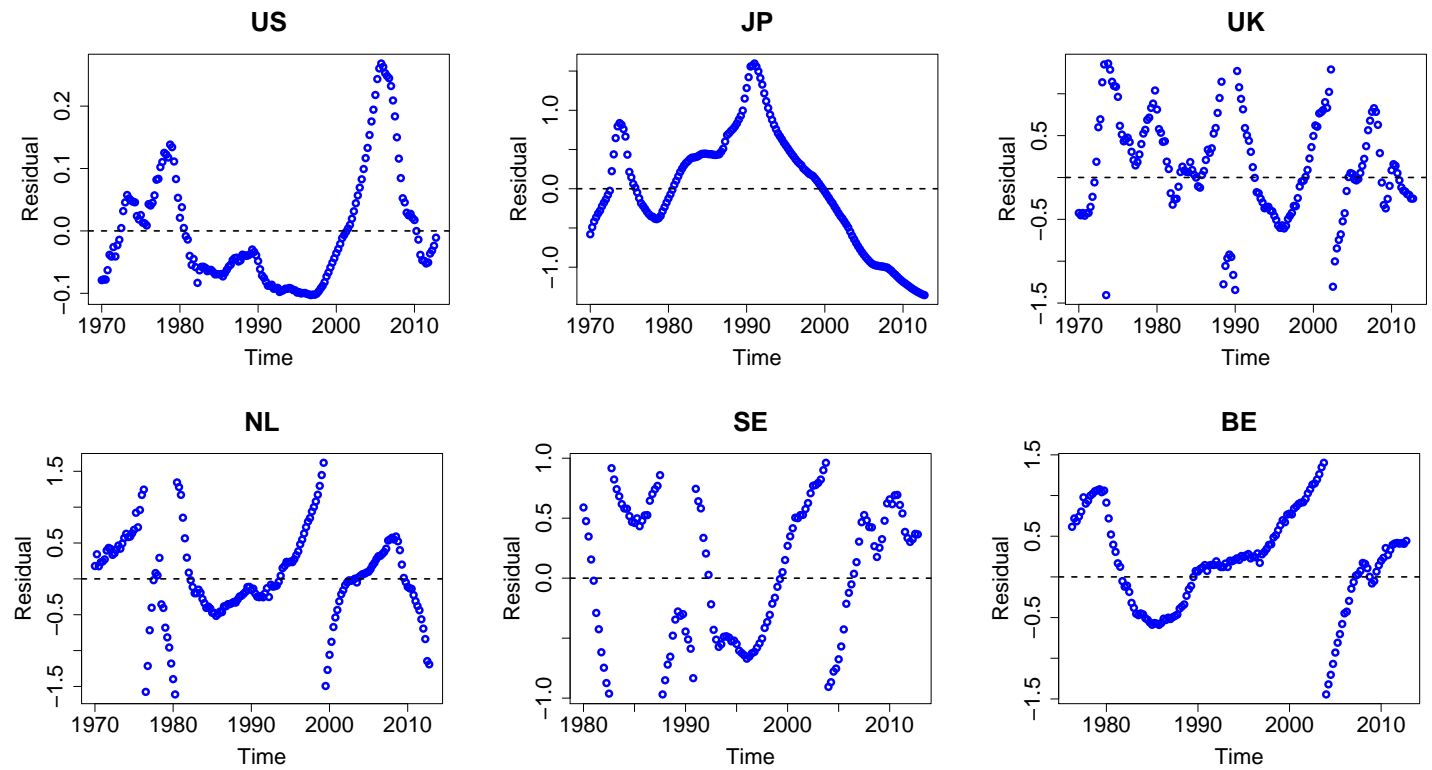

Figure 11: Time series of residuals obtained with Cobb's method. 

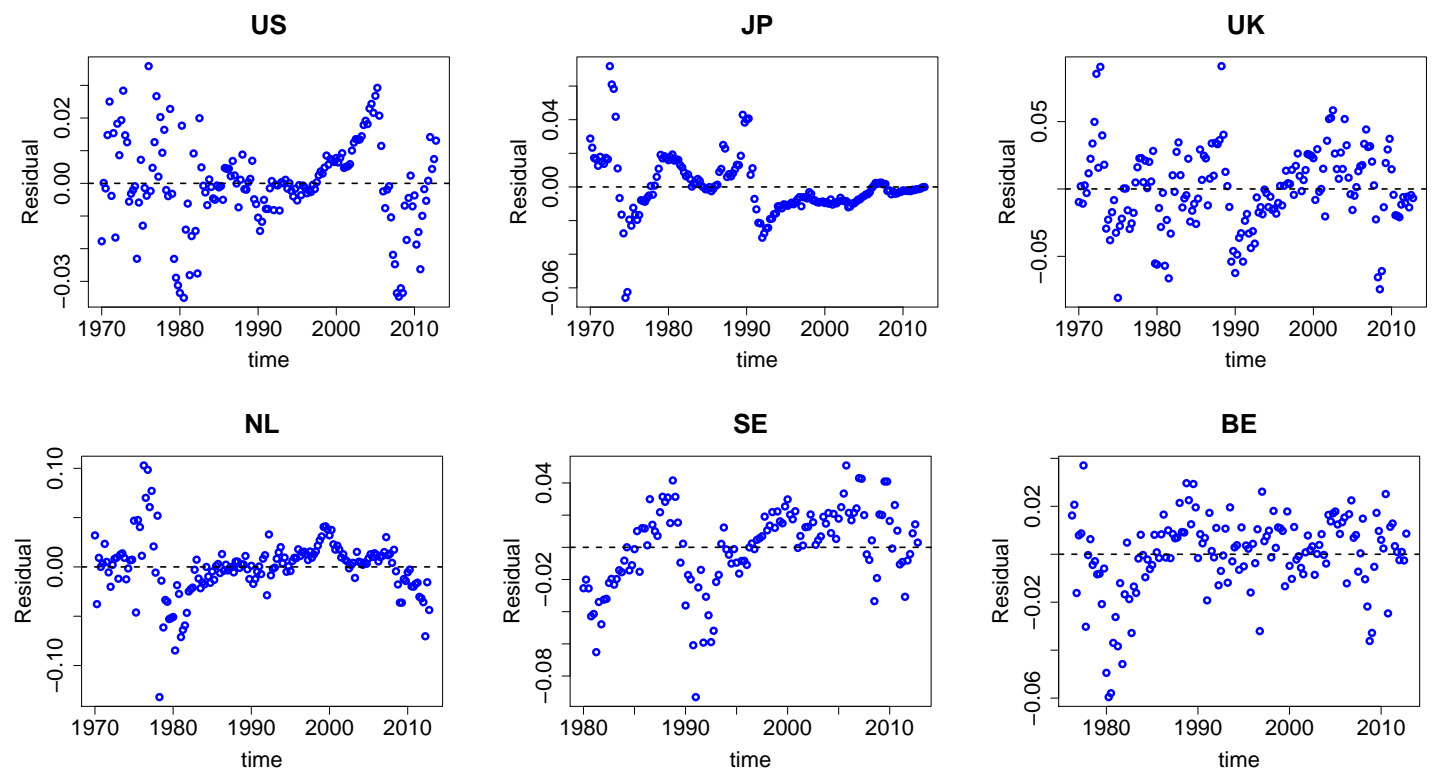

Figure 12: Time series of residuals obtained with Euler discretization.

\section{B Fundamental Price and Price Deviations}

This section provides graphs of the housing price level, the estimated fundamental price, and the relative housing price deviation from the fundamental price, as a function of time (Fig. 13). 

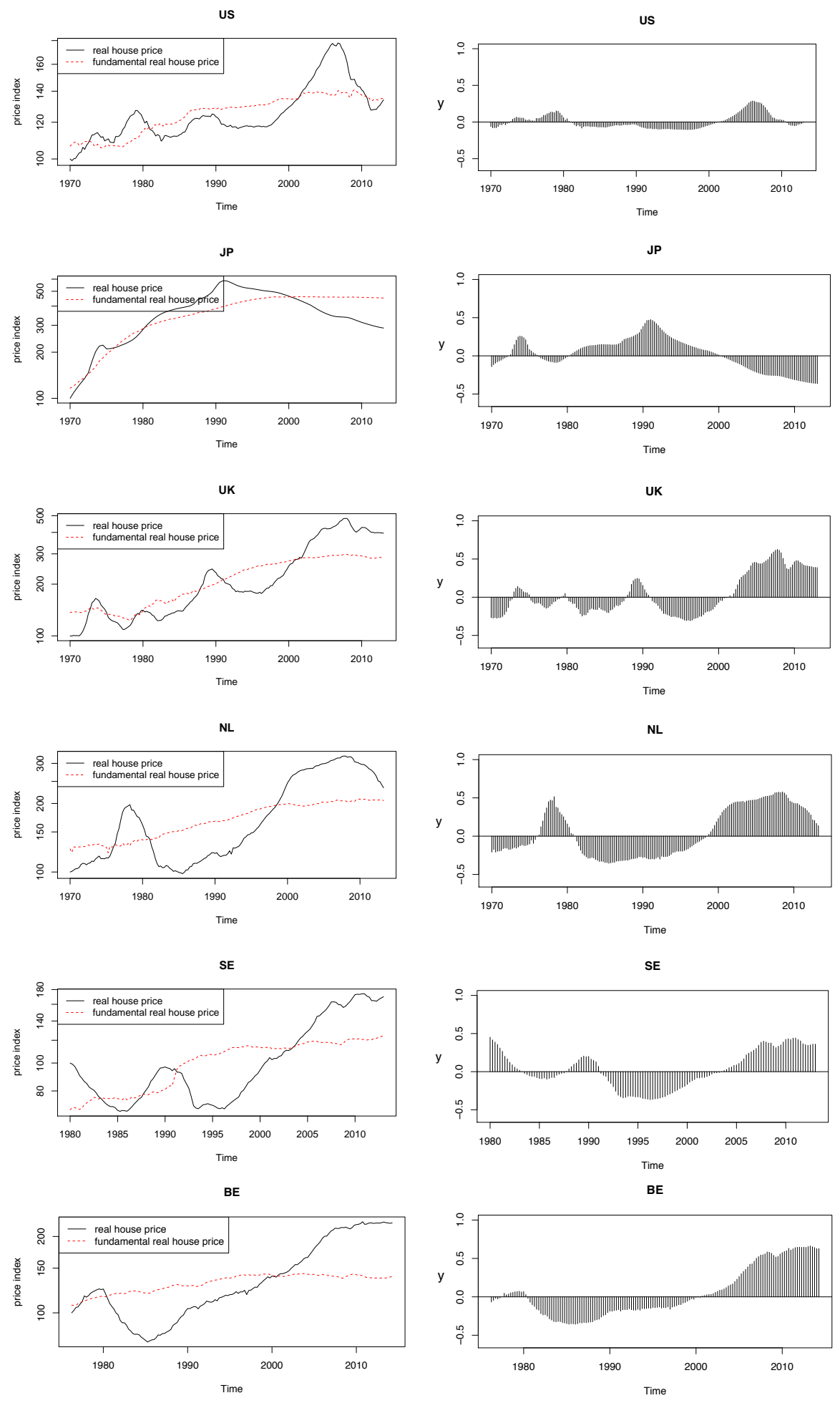

Figure 13: Housing price indices (left, solid lines, 1970Q1=100), estimated fundamental real housing prices (left, dashed lines) and corresponding price deviations $X_{t}$ (right). 